To be published in International Reviews in Physical Chemistry, Vol. 25, issue 4 (Oct-Dec 2006)

\title{
Molecule formation in ultracold atomic gases
}

\author{
Jeremy M. Hutson \\ Department of Chemistry, University of Durham, South Road, Durham, DH1 3LE, England \\ Pavel Soldán \\ Doppler Institute,Department of Physics, Faculty of Nuclear Sciences and Physical Engineering, \\ Czech Technical University, Břehová 7, 11519 Praha 1, Czech Republic
}

(Dated: 21 July 2006)

\begin{abstract}
This review describes recent experimental and theoretical advances in forming molecules in ultracold gases of trapped alkali metal atoms, both by magnetic tuning through Feshbach resonances and by photoassociation. Molecular Bose-Einstein condensation of long-range states of both boson dimers and fermion dimers was achieved in 2002-3. Condensates of boson dimers were found to be short-lived, but long-lived condensates of fermion dimers have been produced. Signatures of triatomic and tetraatomic molecules have recently been observed. Both homonuclear and heteronuclear molecules have been formed by photoassociation, mostly in very high vibrational levels. Recent attempts to produce ultracold molecules in short-range states (low vibrational levels) are described. Experimental and theoretical work on collisions of ultracold molecules is discussed.
\end{abstract}

PACS numbers: 33.80.Ps,34.20.Mq,34.50.Lf,34.50.-s,03.75.Nt,03.75.Ss

\section{Contents}

\section{Introduction}

\begin{tabular}{l} 
Basic properties of ultracold atomic gases \\
\hline Bosons and fermions \\
\hline \hline Hvperfine structure \\
\hline \hline Trapping and cooling \\
\hline Bose-Einstein condensation and Fermi degeneracy \\
\hline \hline Scattering lengths \\
\hline Feshbach resonances \\
\hline
\end{tabular}

Molecules formed by Feshbach resonance tuning 5

Dimers of bosonic atoms

Dimers of fermionic atoms

Heteronuclear Feshbach resonances

Triatomic and larger molecules

\begin{tabular}{|l|}
\hline Molecules formed by photoassociation \\
\hline Photoassociation in Bose-Einstein Condensates \\
\hline \hline Coherent contro] \\
\hline \hline Molecules in low vibrational states \\
\hline
\end{tabular}

Molecules in optical lattices

Collisions of ultracold molecules

Conclusions

Acknowledgments

References

\section{INTRODUCTION}

1 The achievement of Bose-Einstein condensation in 1995 in dilute gases of ${ }^{87} \mathrm{Rb}[1],{ }^{7} \mathrm{Li}[2]$ and ${ }^{23} \mathrm{Na}$ [3] revo-

2 lutionised atomic physics. Since that time, Bose-Einstein

2 condensation has been achieved for several other alkali 2 metal species $\left({ }^{85} \mathrm{Rb}\right.$ 4], ${ }^{41} \mathrm{~K}$ 5] and ${ }^{133} \mathrm{Cs}$ 6] ) and a few 2 other systems $\left({ }^{1} \mathrm{H}\right.$ [7], metastable $\mathrm{He}$ [8, 9], ${ }^{174} \mathrm{Yb}$ [10] 3 and $\left.{ }^{52} \mathrm{Cr} 11\right]$ ). Intense effort has been devoted to the 3 study of the new properties of Bose-Einstein condensates 3 [12, 13, 14, 15]. The field was further broadened by the 4 achievement of quantum degeneracy in Fermi gases of ${ }^{40} \mathrm{~K}\left[16\right.$ ] and ${ }^{6} \mathrm{Li}$ [17], and ultracold fermionic quantum matter has proved to exhibit a new range of novel properties.

Bose-Einstein condensation and Fermi degeneracy in dilute gases typically require temperatures between 1 $\mathrm{nK}$ and $1 \mu \mathrm{K}$. However, new quantum properties start to appear at temperatures around $1 \mathrm{mK}$, where de Broglie wavelengths become large compared to atomic 9 and molecular dimensions. Under these circumstances, 10 collisions are fully quantum-mechanical and are primar11 ily sensitive to long-range interactions. The region below $111 \mathrm{mK}$ is generally referred to as the ultracold regime.

Over the last few years, the focus of research in quan12 tum matter has shifted to the control of ultracold quantum systems. A particularly important development has 13 been the ability to form and manipulate molecules in ultracold atomic gases. Molecules have a much richer

14 energy level structure than atoms, and offer many new possibilities for quantum control. Perhaps most impor-

14 tantly, dipolar molecules interact with one another much more strongly and at longer range than atoms. Dipolar 14 quantum gases are predicted to exhibit more new features 
18. and have possible applications in quantum computing 19]. Cold molecules also have applications in highprecision measurement, and high-resolution spectroscopy on cold molecules may allow the measurement of fundamental physical properties such as the electric dipole moment of the electron [20], the energy differences between enantiomers (which result from parity violation) [21, 22] and the time-dependence of the fine-structure constant [23].

There are two approaches to producing molecular quantum gases: direct approaches, in which pre-existing molecules are cooled from room temperature, and indirect approaches, in which molecules are formed from precooled atoms. The direct approaches have been reviewed previously [24] and there are also several reviews that focus on the applications of scattering theory to directly cooled molecules [25, 26, 27]. The purpose of this article is to review molecule formation in ultracold quantum gases by indirect methods.

\section{BASIC PROPERTIES OF ULTRACOLD ATOMIC GASES}

The physics of cooling and trapping atoms [28, 29, 30, 31 and of the production and properties of BoseEinstein condensates [12, 13, 14, 15] has been reviewed many times. We will restrict ourselves here to a brief discussion, focussing on aspects of the subject that are essential to understanding the present review but may be unfamiliar to readers with a background in physical chemistry rather than atomic physics.

\section{Bosons and fermions}

All fundamental particles are either bosons or fermions. Bosons have integer spin quantum numbers, while fermions have odd half-integer spin quantum numbers. The fundamental difference is encompassed by the Pauli Principle, which states that the total wavefunction for a system must be symmetric with respect to exchange of any pair of identical bosons, but antisymmetric with respect to exchange of a pair of identical fermions. The most important consequence of the Pauli Principle is the Pauli Exclusion Principle, which states that no two fermions in the same spin state can occupy the same spatial state.

Under circumstances where individual electrons cannot be exchanged, atoms and molecules are composite bosons or fermions. Any atom or molecule with an even number of nucleons and electrons is a composite boson and any with an odd number is a composite fermion. For the alkali metals, with an odd number of electrons, isotopes with bosonic nuclei $\left({ }^{6} \mathrm{Li},{ }^{40} \mathrm{~K}\right)$ are composite fermions and isotopes with fermionic nuclei $\left({ }^{7} \mathrm{Li},{ }^{23} \mathrm{Na},{ }^{41} \mathrm{~K}\right.$, etc.) are composite bosons.

\section{Hyperfine structure}

An alkali metal atom with nuclear spin $i$ in its ground electronic state $\left({ }^{2} \mathrm{~S}_{1 / 2}\right)$ can have total angular momentum $f=i \pm \frac{1}{2}$. In a magnetic field, the energy levels split into components labelled by the projection quantum number $m_{f}$, as shown for ${ }^{85} \mathrm{Rb}$ in Fig. 1 (the Breit-Rabi diagram). An atom for which the ground state has the lowest value of $f$ is said to have regular hyperfine structure, and one in which the order is reversed is said to have inverted hyperfine structure. The only alkali metal atom important to the present review that has inverted hyperfine structure is ${ }^{40} \mathrm{~K}$.

The projection quantum number $m_{f}$ is a good quantum number at any field, but $f$ is conserved only at zero field. At fields above the avoided crossings in Fig. 1] $f$ no longer describes the character of the states. In this region the nearly conserved quantities are the individual projections $m_{s}$ and $m_{i}$. This occurs at quite high field for ${ }^{85} \mathrm{Rb}$ but at much smaller fields for atoms with smaller hyperfine splittings such as ${ }^{6} \mathrm{Li}$. Nevertheless, $\left(f, m_{f}\right)$ always provides a unique label for a hyperfine state by following the curve back to low field.

Molecules may have more than one nucleus with nonzero spin, and have mechanical as well as electronic angular momentum. Their energy level structure is thus considerably more complex than for atoms. Nevertheless, it remains true that the zero-field levels are characterised by a total angular momentum $f$, and that in a field these are split into components with projection quantum number $m_{f}$.

\section{Trapping and cooling}

Ultracold atoms and molecules would condense if they came into contact with the walls of a vessel. It is therefore necessary to trap them without physical walls. In most experiments, an atomic beam is first decelerated using light pressure in a Zeeman slower 33], which maintains the atoms in resonance as they slow down. The slow atoms are then confined in a magnetic 34] or magnetooptical [35] trap.

Levels whose energy increases in a magnetic field (see Fig. 1) are known as low-field-seeking, and those whose energy decreases in a field are known as high-field-seeking. A magnetic trap [34] operates by creating a local minimum in the magnetic field strength $B$, so that atoms in low-field-seeking states are trapped. Since it is not possible to create a local maximum in $B$ in free space, high-field-seeking states cannot be trapped magnetically. 


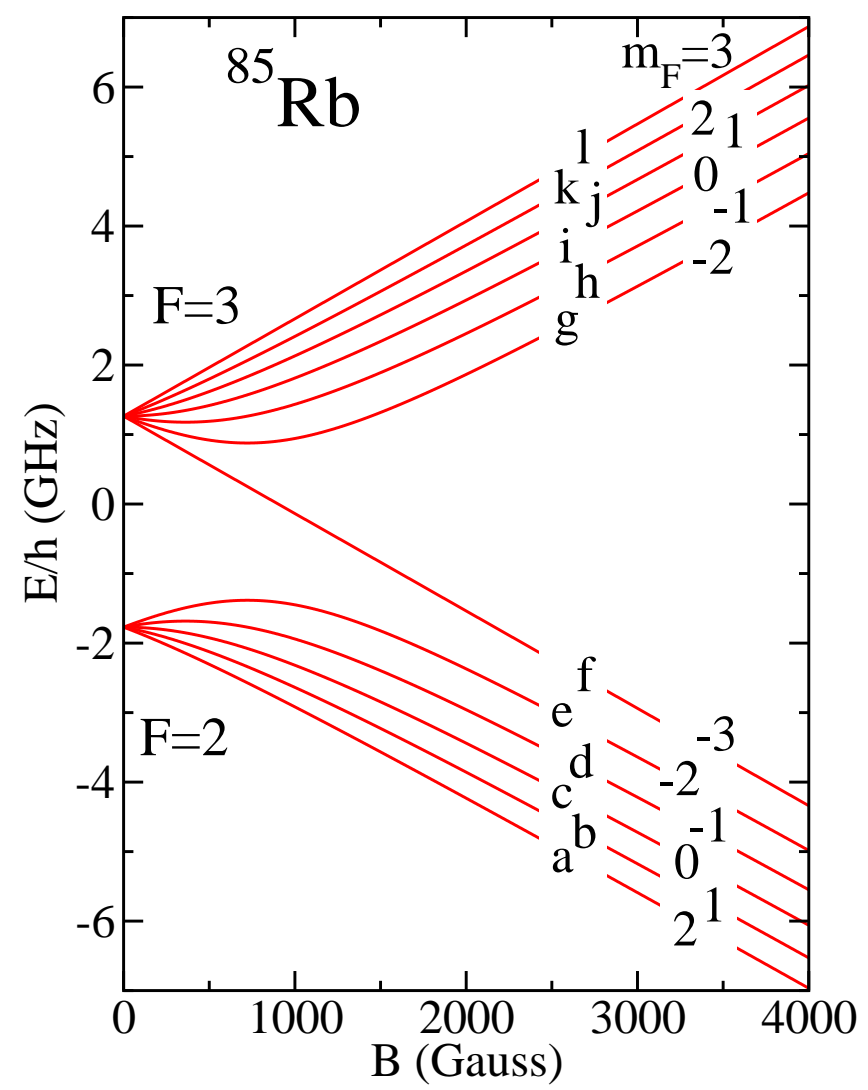

FIG. 1: Zeeman splitting of the hyperfine levels of ${ }^{85} \mathrm{Rb}$ in a magnetic field. ${ }^{85} \mathrm{Rb}$ has $i=5 / 2$ and $f=2$ and 3 . Figure from Tiesinga [32].

For alkali metal atoms, a magnetic trap has a typical depth of around $1 \mathrm{mK}$.

A magneto-optical trap (MOT) 35] uses a combination of magnetic fields and Doppler cooling [36] to trap atoms at considerably higher densities than is possible with magnetic fields alone. A MOT can also cool atoms below the theoretical limit of Doppler cooling by a mechanism known as Sisyphus cooling [37. For example, in early work on ${ }^{23} \mathrm{Na}$, Lett et al. 38. observed a temperature of $40 \mu \mathrm{K}$, compared to the $240 \mu \mathrm{K}$ expected. Even this is not by itself sufficient to achieve Bose-Einstein condensation, and a final stage of cooling, often by evaporative cooling [39], is needed to achieve sub- $\mu \mathrm{K}$ temperatures.

Ultracold atoms can also be confined in an optical dipole trap [40]. Optical dipole traps have the advantage that all magnetic sublevels can be trapped simultaneously. They rely on the fact that the energy of an atom in an electric field oscillating at frequency $\omega$ is $E=-\frac{1}{2} \alpha(\omega) F$, where $F$ is the electric field strength. The frequency-dependent polarizability $\alpha(\omega)$ is positive at low frequencies but is enhanced (and changes sign) near absorption frequencies. An optical dipole trap op- erates by creating an electric field maximum in a region of strong laser radiation, and can be either near-resonant, taking advantage of the enhancement in $\alpha(\omega)$ near an absorption, or far off-resonance. A far off-resonance trap (FORT) [41] causes less heating but is much shallower than a near-resonant trap (sometimes as little as $10 \mu \mathrm{K}$ ). An optical dipole trap in which the laser frequency is so low that the polarizability is close to its static value is referred to as a quasi-electrostatic trap (QUEST) [42].

An optical dipole trap can confine molecules as well as atoms [43]. In addition, molecules with magnetic dipole moments can be trapped magnetically [44, 45]. Molecules with electric dipole moments can be trapped by an analogous electrostatic approach [46].

\section{Bose-Einstein condensation and Fermi degeneracy}

Most traps produce a trapping potential that is nearly harmonic near the minimum. Because of this, the "translational" energy spectrum of trapped atoms is not actually continuous, as is the case for free particles. Instead, it is discretised by the confinement in the trap: the energy level spacings $\hbar \omega$ are typically 10 to $1000 \mathrm{~Hz}$, corresponding to 0.5 to $50 \mathrm{nK}$.

Bosons and fermions follow quite different quantum statistics: Bose-Einstein and Fermi-Dirac statistics respectively. In a Bose-Einstein condensate, nearly all the atoms are in the lowest level in the trap (though there are always uncondensed atoms coexisting with the condensate). In a harmonic trap, condensation occurs at a critical temperature $T_{\mathrm{c}}$ given approximately by $k_{\mathrm{B}} T_{\mathrm{c}}=$ $0.94 \hbar \omega N^{1 / 3}$, where $N$ is the number of atoms [14]. In a Fermi-degenerate gas, by contrast, each level can accommodate only one atom in each spin state $\left(f, m_{f}\right)$, so that the system is characterised by full occupation of trap levels up to the Fermi level, forming a "Fermi sea". The Fermi temperature $T_{\mathrm{F}}$ is again proportional to the level spacing, $k_{\mathrm{B}} T_{\mathrm{F}}=\hbar \omega(6 N)^{1 / 3}$ 14]. Both Bose-Einstein condensation and Fermi degeneracy typically occur at temperatures below $1 \mu \mathrm{K}$.

\section{Scattering lengths}

The scattering wavefunction for collision of a pair of structureless atoms is conveniently written $\psi(r)=$ $r^{-1} \chi(r)$. The radial wavefunction $\chi(r)$ obeys the 1dimensional Schrödinger equation,

$$
\left[-\frac{\hbar^{2}}{2 \mu} \frac{d^{2}}{d r^{2}}+V(r)-E\right] \chi(r)=0
$$

where $E$ is the total energy, $V(r)$ is the effective potential energy and $\mu$ is the reduced mass. For two atoms colliding with zero kinetic energy, $V(r) \rightarrow E$ as $R \rightarrow \infty$, 
so $\chi(r)$ has zero curvature in that region and becomes linear in the interatomic distance $r$,

$$
\chi(r) \sim r-a \text { as } r \rightarrow \infty .
$$

The atom-atom interaction is characterised at the simplest level by the scattering length, $a$, which is the distance at which the continuation of the asymptotic straight line (21) crosses zero.

Many of the properties of a Bose-Einstein condensate depend only on the scattering length and are unaffected by short-range properties of the wavefunction such as the number of nodes. The chemical potential $\mu_{\text {Bose }}$ of a uniform Bose gas is proportional to $a, \mu_{\text {Bose }}=n U_{0}$, where $n$ is the number density, $U_{0}=4 \pi \hbar^{2} a / m$ and $m$ is the atomic mass 14]. A positive scattering length thus corresponds to an interaction that is overall repulsive, while a negative scattering length corresponds to an interaction that is overall attractive. A large Bose-Einstein condensate can usually exist only for positive scattering lengths; for negative scattering lengths there is a limit to the size of condensate that can be formed [47, 48].

The scattering length is closely related to the energy of the highest bound state of the atom-atom pair, $E_{\mathrm{top}}$. If $E_{\text {top }}$ is small and negative (a bound state just below threshold), the scattering length is large and positive and is related to the bound-state energy approximately by

$$
E_{\mathrm{top}}=\frac{-\hbar^{2}}{2 \mu a^{2}} \text {. }
$$

The scattering length increases to infinity as $E_{\text {top }}$ approaches zero and reappears at large negative values when $E_{\text {top }}>0$.

The scattering length depends on the interaction potential and on the reduced mass. It is thus different for pairs of atoms interacting on singlet and triplet curves, and indeed for different hyperfine states. It is also different for different isotopic species.

\section{Feshbach resonances}

A very important discovery was that the interactions between ultracold atoms can be tuned using magnetic fields [49]. As described above, an atom with nuclear spin $i$ in a ${ }^{2} \mathrm{~S}_{1 / 2}$ state can have total angular momentum $f=i \pm \frac{1}{2}$. When two such atoms interact, there are 3 closely-spaced thresholds corresponding to different combinations of hyperfine states as shown in Fig. 2 and there are sets of vibrational levels correlating with each threshold. Some of the high-lying vibrational levels of the upper curves can lie above the lower thresholds. Vibrational levels embedded in a continuum are quasibound and produce Feshbach resonances [50]. In zero field, such resonances are characterised by their energy $E_{\text {res }}$ and width $\Gamma$ (in energy space).

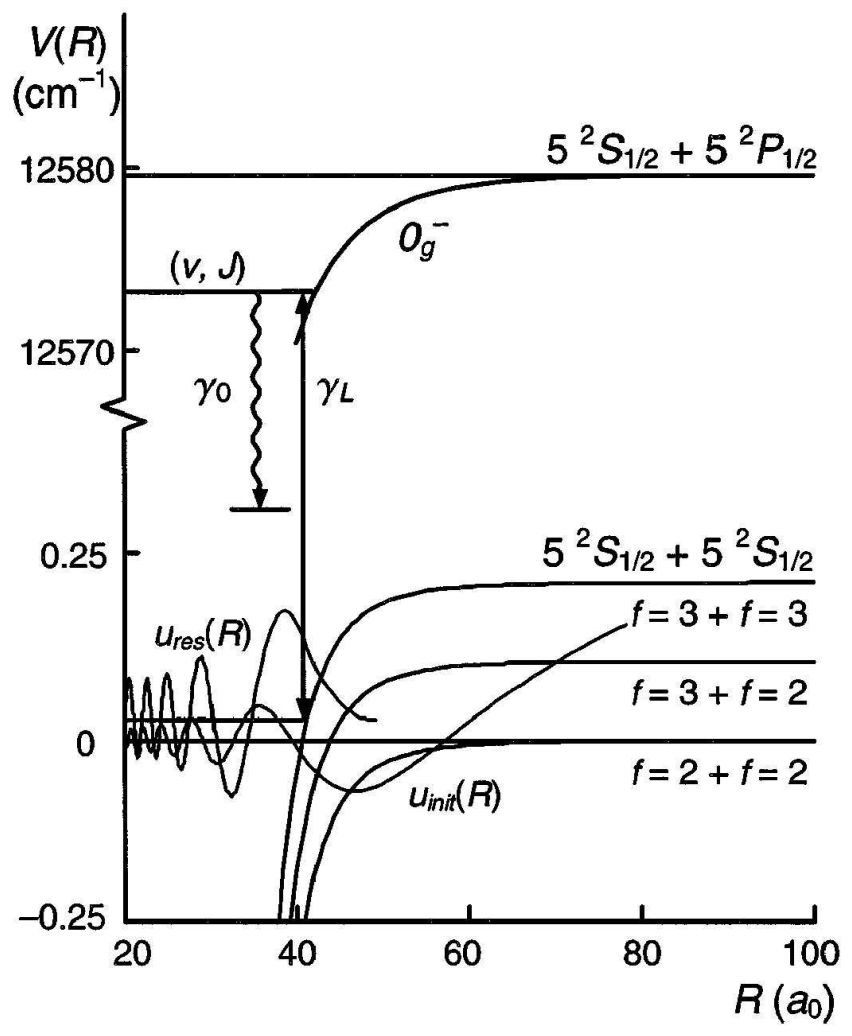

FIG. 2: Potential energy curves for ${ }^{85} \mathrm{Rb}_{2}$ (with nuclear spin $i=5 / 2$ ) showing the 3 hyperfine thresholds. Also shown is a zero-energy scattering wavefunction on the lowest curve $\left(f_{1}, m_{f 1} ; f_{2}, m_{f 2}\right)=(2,-2 ; 2,-2)$ and a bound-state wavefunction on the $(3,-2 ; 3,-2)$ curve that is above the lowest threshold and produces a Feshbach resonance. Reprinted with permission from Courteille et al. [51]. Copyright 1998 by the American Physical Society.

Each combination of atomic quantum numbers produces a channel, and in general there are several channels correlating with each threshold (corresponding to different values of projection quantum numbers $m_{f}$ and values of the quantum numbers $l$ and $m_{l}$ that describe mechanical rotation of the atoms about one another). Each channel has its own potential energy curve, though for simplicity only one curve is shown for each threshold in Fig. 2. At energy $E$, each channel is described as either open or closed (energetically accessible or inaccessible at $R=\infty$ ). For example, at the energy of the state shown in Fig. 2] channels corresponding to the lowest threshold are open and the rest are closed.

In a magnetic field, both the atomic levels (thresholds) and the molecular levels split and shift as shown for ${ }^{87} \mathrm{Rb}$ in Fig. 3 A high-lying vibrational level correlating with one hyperfine state can often be tuned across a lower threshold with an applied magnetic field. In work on ultracold gases, where the collision energy is typically fixed at very near zero and a magnetic field can be varied, 


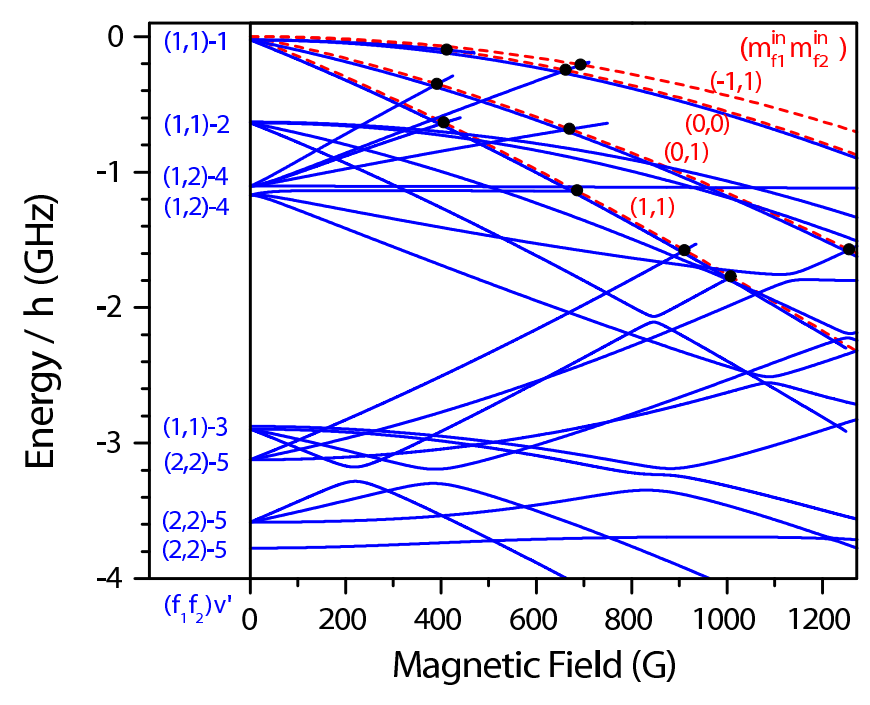

FIG. 3: Tuning of molecular levels (solid lines) and atomic thresholds (dotted lines) for ${ }^{87} \mathrm{Rb}_{2}$ as a function of magnetic field. Feshbach resonances occur at the points marked with filled circles, where a molecular state crosses a threshold. ${ }^{87} \mathrm{Rb}$ has $i=3 / 2$, so there are higher thresholds corresponding to $\left(f_{1}, f_{2}\right)=(1,2)$ and $(2,2)$. Reprinted with permission from Marte et al. 52]. Copyright 2002 by the American Physical Society.

the term "Feshbach resonance" has come to be applied to the behaviour of scattering properties as a function of applied field as a state crosses threshold.

Eq. 3] applies even in the multichannel case, not just when the scattering is governed by a single potential curve. The scattering length thus has a pole whenever there is a bound state at zero kinetic energy, as shown for ${ }^{133} \mathrm{Cs}_{2}$ in the lower panel of Fig. 4. As a function of magnetic field $B$, the scattering length in the vicinity of a Feshbach resonance has the form [53]

$$
a(B)=a_{\mathrm{bg}}\left(1-\frac{\Delta B}{B-B_{0}}\right),
$$

where $a_{\mathrm{bg}}$ is the background scattering length, $B_{0}$ is the resonance position (defined as the field at which $a$ is infinite, which is not quite the same as the field at which the bound state is at zero kinetic energy) and $\Delta B$ is related to the width of the resonance $\Gamma$. In practice, it is of course possible for resonances to overlap or for a sharp resonance to occur in the wings of a broad one. For example, the strong field-dependence of the scattering length for ${ }^{133} \mathrm{Cs}_{2}$ at low magnetic field shown in Fig. 4 can be interpreted in terms of a broad Feshbach resonance at $B_{0}=-8 \mathrm{G}[54]$.

The elastic cross section $\sigma(k)$ for identical bosons at kinetic energy $E_{\text {kin }}=\hbar^{2} k^{2} / 2 \mu$ is approximately given by [58]

$$
\sigma(k)=\frac{8 \pi a^{2}}{1+(k a)^{2}}+\ldots
$$

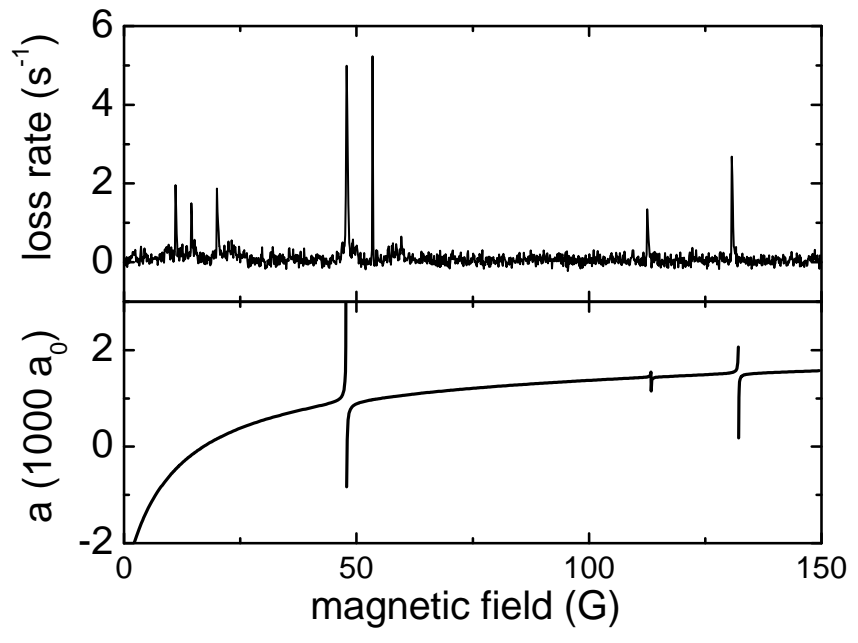

FIG. 4: Feshbach resonances in ${ }^{133} \mathrm{Cs}$ collisions with $\left(f, m_{f}\right)=(3,3)+(3,3)$ as a function of magnetic field. Lower panel: scattering length based on theoretical parameters in ref. 55. Upper panel: loss rate for radiative collisions [56]. Note that some of the sharpest resonances are too narrow to see in the scattering length but are still observed in the loss rates. Figure from Chin [57].

so that $\sigma(k)$ passes through a peak of height $8 \pi / k^{2}$ (corresponding to $a=\infty$ ) at a resonance. Other collisional properties also show sharp features. For example, for ${ }^{133} \mathrm{Cs}$ the radiative loss rate (which is due to atom pairs temporarily excited to the excited electronic state) exhibits sharp peaks as shown in the upper panel of Fig. 4 [55, 56].

Magnetic tuning of Feshbach resonances was first observed by Inouye et al. [59] and Stenger et al. [60], who detected enhanced loss rates from a Bose-Einstein condensate of ${ }^{23} \mathrm{Na}$ as a function of magnetic field, and by Courteille et al. 51], who observed enhanced photoassociation rates in the vicinity of a Feshbach resonance in ${ }^{85} \mathrm{Rb}$. The loss rates were attributed either to excitation of atoms to higher trap states during the field ramp [61, 62] or to inelastic collisions involving the transiently formed molecular state 63]. In experiments on ${ }^{85} \mathrm{Rb}$, Roberts et al. [64] and Donley et al. 65] demonstrated that tuning the scattering length suddenly from positive to negative could cause controlled collapse of a Bose-Einstein condensate. The collapse produces an explosion of atoms from the condensate and has come to be known as a Bosenova by analogy with astrophysical supernovae.

\section{MOLECULES FORMED BY FESHBACH RESONANCE TUNING}

The possibility of using Feshbach resonances to create ultracold molecules was first predicted in 1999 61, 


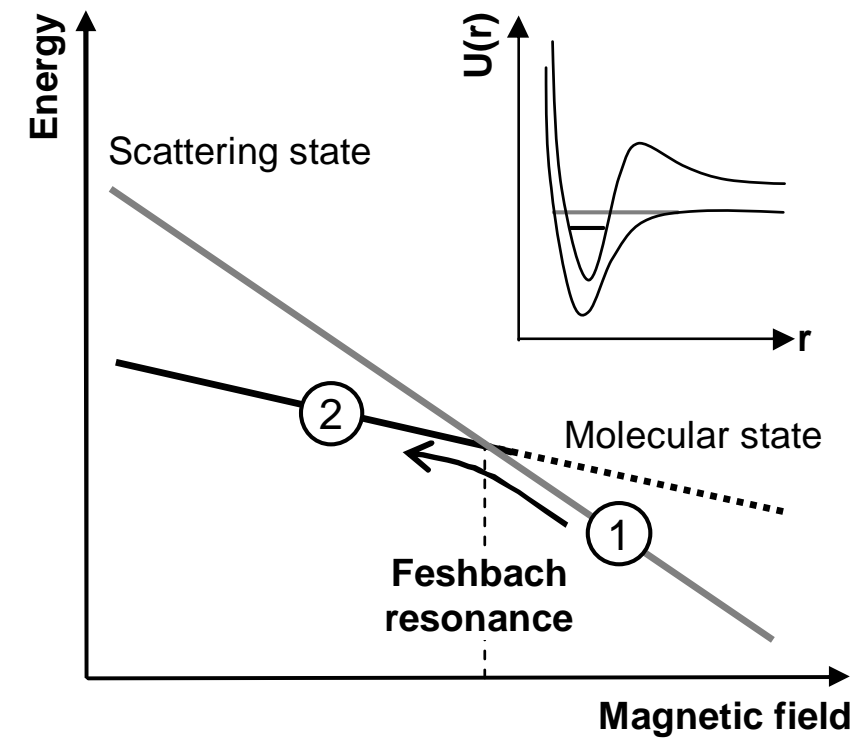

FIG. 5: The crossing of atomic (scattering) and molecular states as a function of magnetic field, showing the avoided crossing and the use of a field ramp to convert pairs of atoms into molecules. Note that in this case the atomic and molecular states are both high-field-seeking, so cannot be trapped magnetically. Reprinted with permission from Herbig et al. 67]. Copyright 2003 by Macmillan Publishers Ltd.

The actual arrangement of states in a Feshbach resonance is sometimes the mirror image of that shown in Fig. [5 In the following discussion, the side of the resonance where molecules are lower in energy than atoms will be referred to as the molecular side of the resonance.

\section{Dimers of bosonic atoms}

The first signatures of trapped molecules produced by Feshbach resonance tuning were observed by Donley et al. [68] in 2002. They worked with magnetically trapped ${ }^{85} \mathrm{Rb}$, so were restricted to low-field-seeking states. ${ }^{85} \mathrm{Rb}$ has nuclear spin $i=5 / 2$, and Donley et al. worked with trapped atoms in the $\left(f, m_{f}\right)=(2,-2)$ state, which is not the lowest state in a magnetic field (see Fig. 1). In this case the energy levels are the mirror image of those shown in Fig. 5 with the molecular state below the atomic state on the high-field side of the resonance.
The experiment of Donley et al. was a little more complicated than a simple ramp over the resonance as shown in Fig. 5 Since the background scattering length for this system is negative, $a(B)$ is negative on the atomic side even far from the resonance. For this reason it was not possible to approach the resonance from the atomic side, because the condensate collapsed [64, 65]. Donley et al. therefore used a magnetic field profile that approached the Feshbach resonance from the molecular side but did not actually cross it. The fast field ramp mixed the atomic and molecular states, and Donley et al. observed quantum beats (Ramsey fringes) between trapped atoms and molecules.

Over the following two years, the technique was developed by several groups and extended to other bosonic systems: ${ }^{133} \mathrm{Cs}_{2}$ [67], ${ }^{87} \mathrm{Rb}_{2}$ [69] and ${ }^{23} \mathrm{Na}_{2}$ [70. In all these experiments atoms were prepared in the lowest hyperfine state in a magnetic field, with $f=i-1 / 2$ and $m_{f}=f$. Such states cannot be trapped magnetically (because they are high-field-seeking), so the atoms were confined in optical traps. Molecules were created by sweeping the magnetic field across a resonance as shown in Fig. 5 The molecules were separated from the remaining atoms either magnetically [67, 69] or by using a laser resonant with the atoms but not the molecules to push the atoms out of the trap [70]. The molecules were then detected and imaged by converting them back to atoms with a reverse field sweep. Fig. [6 shows images of the $\mathrm{Cs}_{2}$ molecular cloud created by Herbig et al. 67].

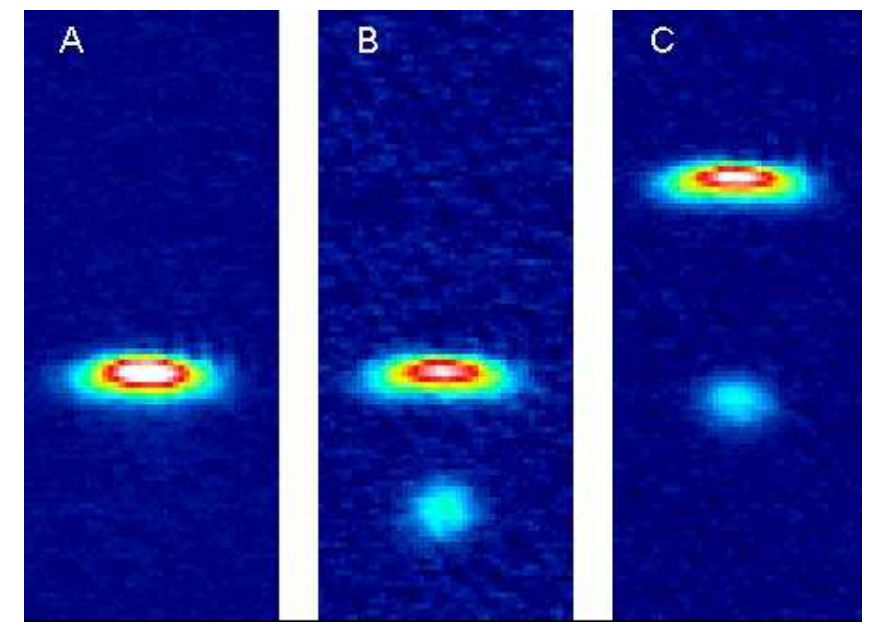

FIG. 6: Formation of a quantum gas of ${ }^{133} \mathrm{Cs}_{2}$ molecules. A: magnetically levitated atomic BEC; B: levitated atomic BEC with falling molecular cloud below; C: levitated molecular cloud with rising atomic BEC above. Reprinted with permission from Herbig et al. 67]. Copyright 2003 by Macmillan Publishers Ltd.

In all these experiments involving bosonic atoms it was found that the molecules were lost from the trap within a few milliseconds. The fast trap loss was attributed to 
atom-diatom collisions. The molecules are formed in a very highly excited state, often the highest vibrational state that exists in the potential well. There are always many lower-lying states, and even near dissociation the vibrational spacing is large compared to the depth of the trap. Thus inelastic atom-molecule collisions,

$$
\mathrm{M}_{2}(v)+\mathrm{M} \longrightarrow \mathrm{M}_{2}\left(v^{\prime}<v\right)+\mathrm{M}
$$

or molecule-molecule collisions,

$$
\mathrm{M}_{2}(v)+\mathrm{M}_{2}(v) \longrightarrow \mathrm{M}_{2}\left(v^{\prime}<v\right)+\mathrm{M}_{2}\left(v^{\prime \prime} \leq v\right),
$$

always release enough kinetic energy to eject both collision partners from the trap. It should be noted that the molecules are not destroyed in such collisions, but they are lost from the trap and are no longer ultracold.

As remarked above, the molecules produced by Donley et al. [68] are formed from ${ }^{85} \mathrm{Rb}$ atoms in the $\left(f, m_{f}\right)=$ $(2,-2)$ state, which is not the lowest state in a magnetic field. They are thus not strictly bound, and can dissociate spontaneously without collisions to form atoms in lower-lying hyperfine states. This has been studied experimentally by Thompson et al. 71] and theoretically by Köhler et al. [2]. Thompson et al. 71] adapted the experiment of Donley et al. 68] to create ${ }^{85} \mathrm{Rb}_{2}$ molecules using a magnetic field sweep through the Feshbach resonance without holding the atomic condensate at $a<0$ for long enough for it to collapse. As in the earlier experiments, the molecules were formed in states above the lowest hyperfine threshold. Even at densities where collisional loss was very slow, Thompson et al. found that the molecules decayed within $1 \mathrm{~ms}$ at magnetic fields far from resonance. However, close to resonance, they were able to achieve lifetimes of tens of milliseconds. It should be noted that this decay mechanism is not applicable to the experiments on ${ }^{133} \mathrm{Cs}_{2},{ }^{87} \mathrm{Rb}_{2}$ and ${ }^{23} \mathrm{Na}_{2}$ [67, 69, 70], where the molecules are formed in states that lie below the lowest atomic threshold.

Molecules formed by Feshbach resonance tuning are very large. Even the closed-channel part of the wavefunction corresponds to a molecule in a very high vibrational state, as shown in Fig. 2 the wavefunction peaks near the outer turning point, which can be at distances approaching $R=100 a_{0}$. However, even this underestimates the size of Feshbach molecules. Köhler et al. 73. have investigated a model of the Feshbach resonance used to produce ${ }^{85} \mathrm{Rb}_{2}$ by Donley et al. [68], and have shown that for magnetic fields near the resonance the molecular wavefunction is dominated by the open (atomic) channel and has a wavefunction that dies off as $\exp (-r / a)$ at long range; the mean internuclear distance is on the order of $a / 2$, which near a resonance can be several thousand $a_{0}$. An example of this is shown in Fig. 7

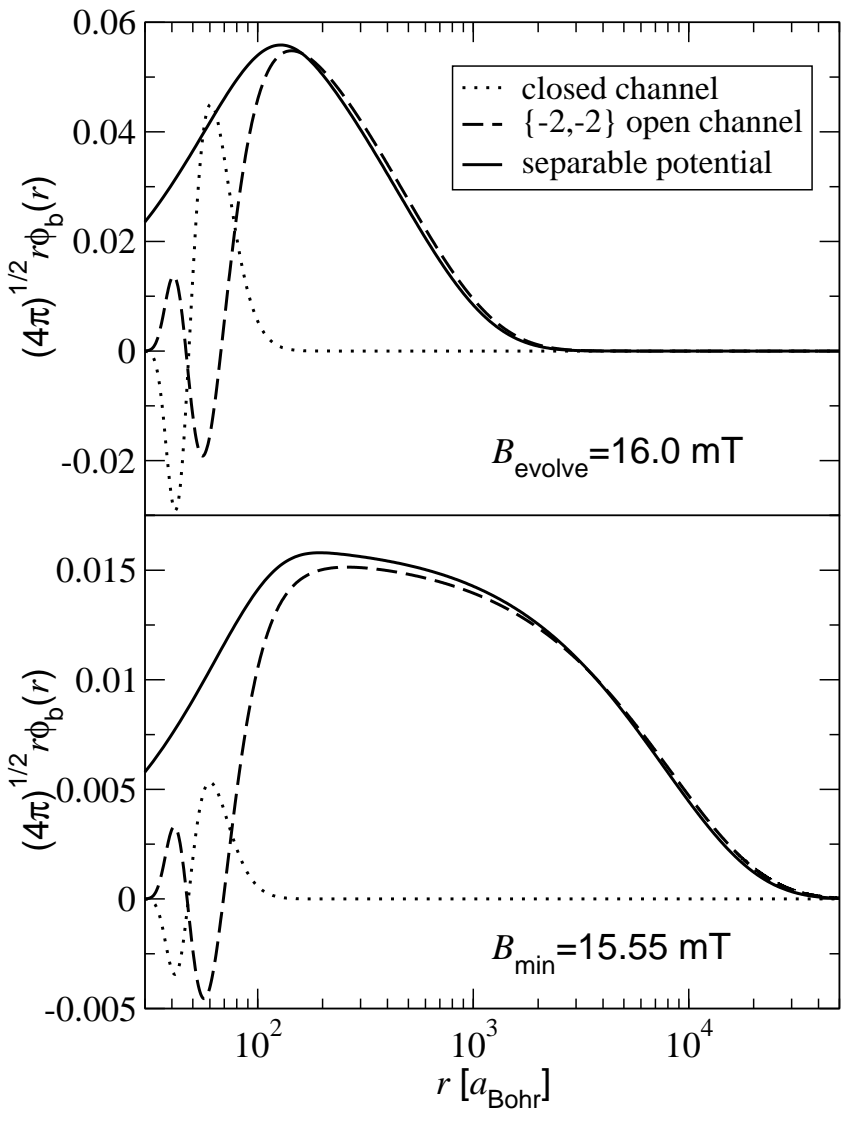

FIG. 7: Bound-state wavefunctions for a model of ${ }^{85} \mathrm{Rb}_{2}$ close to a Feshbach resonance $\left(B=15.55 \mathrm{mT}, a=7800 a_{0}\right)$ and slightly further away $\left(B=16.0 \mathrm{mT}, a=521 a_{0}\right)$. Reprinted with permission from Köhler et al. 73]. Copyright 2003 by the American Physical Society.

\section{Dimers of fermionic atoms}

In parallel to the work on boson dimers, molecules were created from pairs of fermionic atoms by tuning through Feshbach resonances. Collision rates for two identical fermions are suppressed because s-wave scattering (partial wave $l=0$ ) is forbidden. Accordingly, the fermion work focussed on pairs of atoms in different spin states, again in optical traps. Molecule formation was first achieved for ${ }^{40} \mathrm{~K}_{2}$ by Regal et al. [74]. ${ }^{40} \mathrm{~K}$ has nuclear spin $i=4$ and an inverted hyperfine structure, so that the atomic ground state in a magnetic field has $\left(f, m_{f}\right)=(9 / 2,-9 / 2)$. Regal et al. used a mixture of $(9 / 2,-9 / 2)$ and $(9 / 2,-5 / 2)$ atoms and formed molecules by magnetic tuning through a Feshbach resonance. Once again the molecules were found to be short-lived $(\tau \approx 1$ $\mathrm{ms})$. The lifetime was attributed to vibrationally inelastic collisions 74], though spontaneous dissociation by spin relaxation is also possible in this case.

A major experimental breakthrough came in mid-2003, when four groups [75, 76, 77, 78] independently reported 
within a period of 6 weeks that fermion dimers can be remarkably stable to collisions when the atom-atom scattering length is tuned to a large positive value. ${ }^{6} \mathrm{Li}$ has nuclear spin $i=1$, and Strecker et al. 75 and Cubizolles et al. 76 . prepared ${ }^{6} \mathrm{Li}_{2}$ molecules by Feshbach resonance tuning in mixtures of the lowest two spin states, correlating with $\left(f, m_{f}\right)=(1 / 2,1 / 2)$ and $(1 / 2,-1 / 2)$ at low field. Both groups showed that the molecules remained trapped for $1 \mathrm{~s}$ or more before being dissociated by a reverse magnetic field sweep. Jochim et al. 77] prepared ${ }^{6} \mathrm{Li}_{2}$ molecules by a different method, taking advantage of the dramatically enhanced 3-body recombination rate near a Feshbach resonance, and observed comparable lifetimes. Cubizolles et al. [6] and Jochim et al. 77] showed that the lifetime was particularly large close to the resonance, where the scattering length is large and positive. Regal et al. 78] carried out analogous experiments on ${ }^{40} \mathrm{~K}_{2}$, both for the spin states involved in their earlier experiments 74] and for molecules formed from $\left(f, m_{f}\right)=(9 / 2,-9 / 2)$ and $(9 / 2,-7 / 2)$. They confirmed the fast decay far from resonance, but showed that for large positive scattering lengths the lifetime was dramatically enhanced.

By the end of 2003, three different groups had succeeded in creating long-lived molecular Bose-Einstein condensates of fermion dimers. Jochim et al. [79] and Zwierlein et al. 80] achieved this by evaporative cooling combined with 3 -body recombination in a mixed gas of ${ }^{6} \mathrm{Li}$ in its $\left(f, m_{f}\right)=(1 / 2,1 / 2)$ and $(1 / 2,-1 / 2)$ states, held at large positive scattering length close to a Feshbach resonance. Under these circumstances 3-body recombination to form molecules is thermodynamically favourable and the molecules are long-lived. Greiner et al. 81] formed ${ }^{40} \mathrm{~K}_{2}$ from a very cold Fermi gas of ${ }^{40} \mathrm{~K}_{2}$ atoms in their $\left(f, m_{f}\right)=(9 / 2,-9 / 2)$ and $(9 / 2,-7 / 2)$ states, using a Feshbach ramp so slow that thermal equilibrium was maintained during the field sweep. All three groups observed the sudden appearance of a sharp spatial peak in the density with decreasing temperature, as shown in Fig. 8 for ${ }^{40} \mathrm{~K}_{2}$. This is widely regarded as the "smoking gun" of Bose-Einstein condensation 1, 3].

Petrov et al. 82, 83 analysed the stability of fermion dimers in terms of the long-range form of the wavefunction. In the case where the atom-atom scattering length $a$ is much larger than the range of the atom-atom potential $r_{e}$, they showed that both the atom-molecule and molecule-molecule inelastic collision rates are suppressed by Fermi statistics. However, their derivation applies only to molecules that are in long-range states, with a wavefunction that depends on the scattering length, $\chi(r) \sim \exp (-r / a)$. As will be discussed in more detail below, Cvitaš et al. [84] have shown computationally that there is no systematic suppression of the atom-molecule inelastic rate for fermion dimers in low-lying vibrational levels, even when $a$ is large and positive.

A major reason for interest in fermion dimers is that a
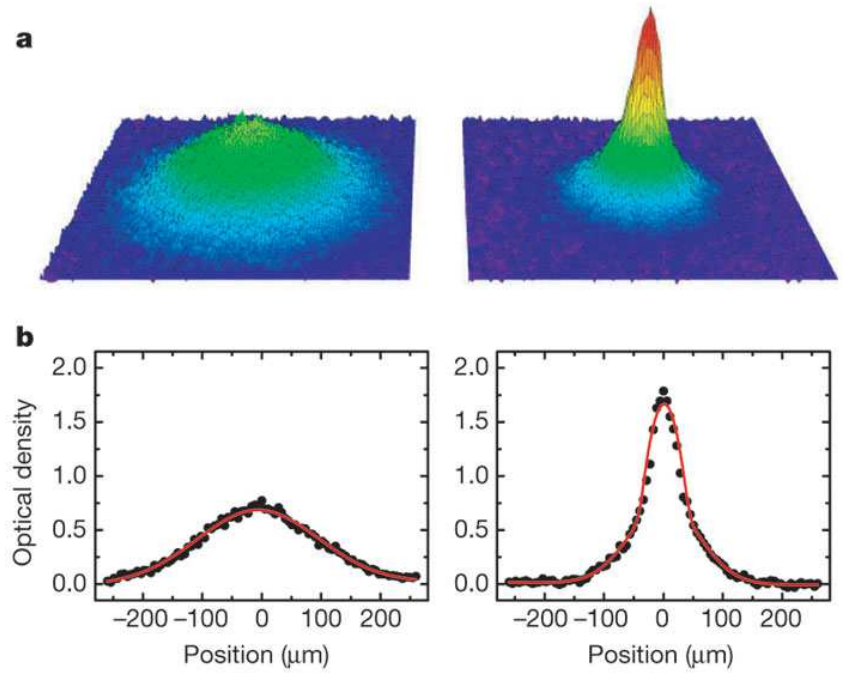

FIG. 8: Images of a molecular cloud of ${ }^{40} \mathrm{~K}_{2}$ after $20 \mathrm{~ms}$ of free expansion, above and below the critical temperature for BoseEinstein condensation. The condensed cloud (right) shows the tight spatial peak characteristic of a condensate. Reprinted with permission from Greiner et al. 81]. Copyright 2003 by [check organisation]

they provide tunable models for studying problems in condensed matter physics such as the origin of superfluidity and superconductivity. According to the widely accepted theory of Bardeen, Cooper and Schrieffer (BCS) [85], superconductivity occurs because pairs of electrons are composite bosons and can therefore condense. However, the pairs of electrons involved in superconductivity (Cooper pairs) are much larger than the mean separation between electrons. This is different from the usual regime of molecular Bose-Einstein condensation (BEC), because far from a resonance a fermion dimer is relatively strongly bound and is small compared to the typical separation between unbound atoms. However, close to resonance the "size" of the molecules increases and can become comparable to the atom-atom spacing. Under these circumstances the "molecules" are interpenetrating and lose their identity in very much the same way as Cooper pairs of electrons. The beauty of the fermionic atom systems is that this transition can be followed as a function of magnetic field.

The BEC-BCS crossover between a molecular BoseEinstein condensate and a condensate of atomic Cooper pairs has been studied extensively. The BCS regime is an intrinsically many-body regime in which 2-body theories cannot be expected to work. At the centre of a resonance the 2-body scattering length is infinite but the pair size in a many-body system is limited to the atomatom spacing. Beyond the resonance, where the scattering length is negative, the binding forces are much smaller and the pair size becomes much larger then the interparticle spacing. Bartenstein et al. [86] showed that 
the transition between the two regimes can be achieved smoothly and reversibly. Regal et al. 87, 88 and Zwierlein et al. 89. demonstrated Bose-Einstein condensation of Cooper pairs in the BCS regime and measured the atomic momentum distribution in the BCS region. Chin et al. [90] and Greiner et al. 91] observed a pairing gap characteristic of superfluidity. Partridge et al. 92 have measured the closed-channel component of the pair wavefunction on both sides of a resonance and shown that, though small, it persists on the BCS side of the resonance, contrary to the predictions of 2-body theory.

Other studies have focussed on signatures of superfluidity rather than Cooper pairing. Kinast et al. 93] and Bartenstein et al. 94 observed collective oscillations in a strongly interacting Fermi gas that suggest superfluid behaviour in the BCS regime, while Kinast et al. 95. measured the heat capacity of such a gas. Most recently, Zwierlein et al. [96] provided conclusive evidence of superfluidity by observing arrays of vortices on both the BEC and BCS sides of a resonance in ${ }^{6} \mathrm{Li}_{2}$.

\section{Heteronuclear Feshbach resonances}

It is possible to trap two different alkali metal species simultaneously, and magnetic Feshbach resonances have been observed in RbK 97, 98] and LiNa 99]. There is little doubt that tuning through such resonances will soon be used to form heteronuclear molecules. However, it should be noted that neutral heteronuclear molecules in long-range states do not have significant dipole moments: typical values are less than $0.3 \mathrm{D}$ at $R=15 a_{0}$ [100] and decay as $D_{7} R^{-7}$ at long range [101].

\section{Triatomic and larger molecules}

It is in principle possible to form molecules larger than diatomic, either by direct association from atoms or by association of smaller molecules. Chin et al. 102 have formed $\mathrm{Cs}_{2}$ molecules by Feshbach resonance tuning and then separated out the remaining atoms magnetically. They observed field-dependent resonances in the inelastic loss rates that they attributed to $\mathrm{Cs}_{4}$ bound states near the molecular scattering threshold.

For three atoms there is the intriguing prospect of forming Efimov states 104], which are long-range trimer states that exist even when the corresponding dimers are unbound. Indeed, Efimov showed that, if the pair potential has exactly one bound state at zero energy (corresponding to an infinite scattering length), there are an infinite number of such trimer states. The helium trimer is predicted to have one Efimov state [105, 106], but it has not yet proved possible to observe this experimentally. However, alkali metal atoms with tunable interactions offer new possibilities for a slightly different type

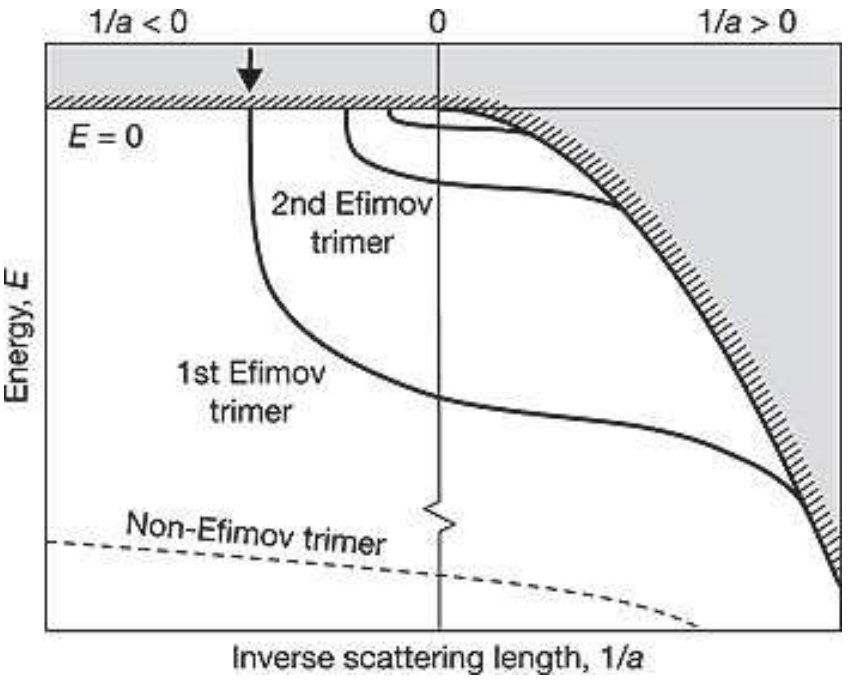

FIG. 9: Appearance of Efimov trimer states, showing how they intersect the threshold for 3 separated atoms as a function of scattering length. The shaded area shows the scattering continuum for 3 atoms $(a<0)$ and for atom + diatom $(a>0)$. The characteristic factor of 22.7 in $a$ has been reduced to 2 for the purpose of illustration. Reproduced from Kraemer et al. 103].

of Efimov state, which occurs whenever the scattering length is large but is complicated by the existence of a large number of deeply bound states. Braaten and Hammer [107] and Nielsen et al. 108] have shown that Efimov states will cause resonant enhancements in 3-body recombination rates at characteristic values of the scattering length that differ by successive factors of 22.7. The characteristic dependence of energies on scattering length is shown in Fig. 9 Very recently, Kraemer et al. 103] have measured trap loss in an ultracold gas of Cs atoms as a function of scattering length, and observed a peak that they attribute to resonance between an Efimov state of the trimer and the threshold for 3 separated atoms.

\section{MOLECULES FORMED BY PHOTOASSOCIATION}

Dimers can also be formed in cold atomic gases by photoassociation, as predicted by Thorsheim et al. in 1987 [109]. Developments up to 1999 were reviewed by Stwalley and Wang [110] and more recent work by Jones et al. 111]. The early work focussed on 1-photon photoassociation spectroscopy, forming molecules in electronically excited states. More recently, however, it has become possible to form ultracold molecules in their electronic ground states by 2-photon processes as shown in Fig. 10. This was first achieved by Fioretti et al. 112], who photoassociated $\mathrm{Cs}_{2}$ to an excited $0_{g}^{-}$electronic state with a double minimum [113] and observed ultracold molecules 
formed by spontaneous emission to the lowest triplet state. Nikolov et al. [114] carried out a similar experiment to form the ${ }^{1} \Sigma_{g}^{+}$ground state of $\mathrm{K}_{2}$ and used resonant two-colour ionization to show that the vibrational distribution peaked at $v=36$, just over half way up the ground state potential well. They subsequently 115 developed a 2-photon excitation scheme via an excited ${ }^{1} \Pi_{u}$ state that produced molecules even further down the ground-state well. Optical trapping of ground-state $\mathrm{Cs}_{2}$ molecules was achieved by Takekoshi et al. [43] and magnetic trapping by Vanhaecke et al. [45].

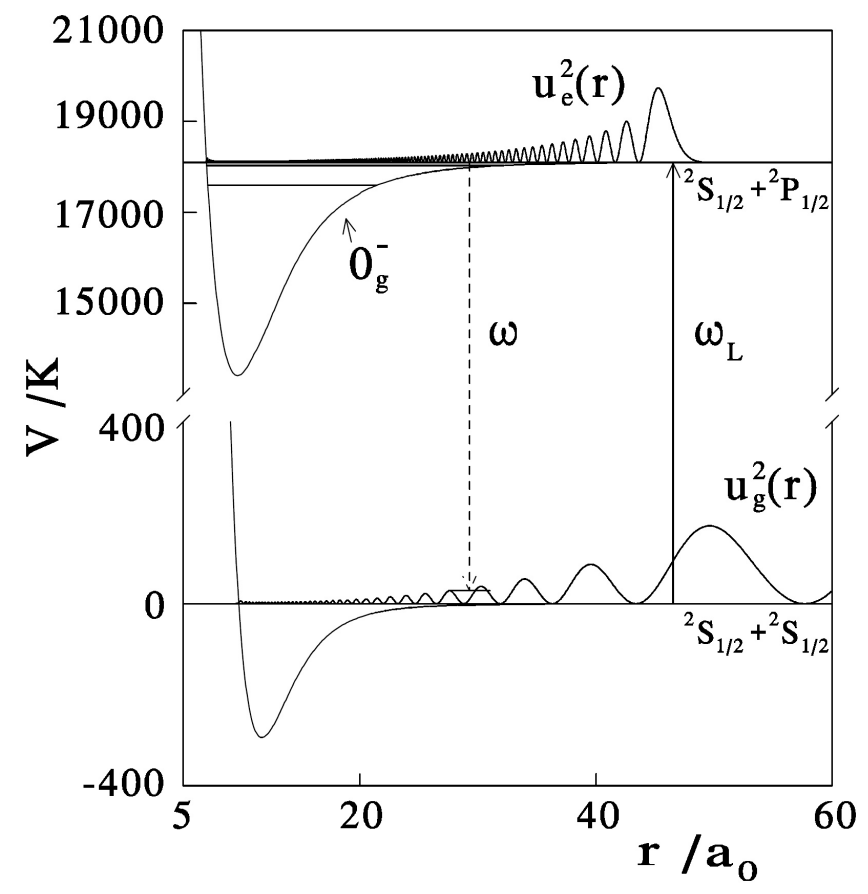

FIG. 10: Potential energy curves and (squares of) vibrational wavefunctions for photoassociation of $\mathrm{Rb}_{2}$. Reprinted with permission from Boesten et al. [116]. Copyright 1999 by Institute of Physics.

\section{Photoassociation in Bose-Einstein Condensates}

An additional degree of control can be introduced by using stimulated Raman adiabatic passage (STIRAP) [17], in which a second laser detuned from the excitation laser brings the molecules down to bound levels of the ground electronic state [118]. This was first achieved by Wynar et al. [119], who worked in a Bose-Einstein condensate of ${ }^{87} \mathrm{Rb}$ and produced ultracold ${ }^{87} \mathrm{Rb}_{2}$ molecules in a specific vibration-rotation (and hyperfine) state by STIRAP via the $0_{g}^{-}$state. In their experiment the dump laser was detuned by only $636 \mathrm{MHz}$ from the pump laser, and the molecules were formed in the second-to-last vibrationally excited state. Producing molecules in this way has the major advantage that coherence can be maintained 120, 121]; this is not the case if spontaneous emission is involved. Winkler et al. 122 have used 2colour photoassociation to produce a coherent superposition of atomic ${ }^{87} \mathrm{Rb}$ and molecular ${ }^{87} \mathrm{Rb}_{2}$ Bose-Einstein condensates. In a conceptually related but experimentally quite different approach, Thompson et al. [123] have used an oscillating magnetic field to stimulate deexcitation of atom pairs from free atom states to molecular states of ${ }^{85} \mathrm{Rb}_{2}$.

1-photon and coherent 2-photon photoassociation can be viewed as manifestations of optical Feshbach resonances, in which laser frequencies or intensities are used to tune collision properties. In a "dressed state" picture, the photons of a laser field bring atomic or molecular states at different energies into resonance with one another. Such resonances were first investigated theoretically by Fedichev et al. 124], who showed that moderate laser intensities near resonance could induce sufficient excited-state character to change scattering lengths and even reverse their sign. Bohn and Julienne 125] extended this work and introduced the concept of 2-photon (or 2colour) optical Feshbach resonances, in which the state responsible for the resonance is a vibrational level of the ground electronic state, connected to the atomic state by a 2-photon (stimulated Raman) transition.

Optical Feshbach resonances were first demonstrated experimentally by Fatemi et al. [126], who observed changes in Na-Na scattering properties due to a onephoton resonance as a function of laser detuning and intensity. Theis et al. [127] observed the variation of scattering length directly, by using Bragg spectroscopy to determine the mean field energy of an ${ }^{87} \mathrm{Rb}$ condensate, and Thalhammer et al. 128] have carried out similar measurements for a stimulated Raman resonance.

An optical Feshbach resonance can in principle be used to create molecules in a very similar way to a magnetic Feshbach resonance. Javanainen and Mackie 129] proposed a photoassociation scheme in which molecules are produced coherently by chirping (ramping) the laser frequency adiabatically across an optical Feshbach resonance. Koch et al. 130 have investigated the formation of electronic ground-state ${ }^{87} \mathrm{Rb}_{2}$ molecules in a similar way, and considered ramping the laser intensity as well as the frequency.

Tuning through optical Feshbach resonances is potentially more general than magnetic tuning. Laser fields can be switched on and off much faster than magnetic fields, and optical tuning could be applied to atoms without nuclear spin, such as the predominant isotopes of several of the alkaline earths $\left({ }^{24} \mathrm{Mg},{ }^{40} \mathrm{Ca},{ }^{88} \mathrm{Sr},{ }^{138} \mathrm{Ba}\right)$. In addition, levels can be tuned into resonance from much further away using 2-photon resonances than is possible magnetically. However, a limitation arises because the extent to which the crossing is avoided depends on the laser-induced coupling between the two states, and thus 
on transition moments and Franck-Condon factors. If the Raman transition is very weak, it will be impossible to tune across the resonance slowly enough to achieve adiabatic passage.

\section{Coherent control}

Most photoassociation experiments have so far used fixed-frequency lasers. However, an alternative is to use laser pulses with tailored frequency and intensity profiles, as has become common in quantum control experiments on molecules at higher temperature [131]. A broadband laser can create a non-stationary state (wavepacket) that is made up of a linear combination of several different rovibrational levels of the excited electronic state. The wavepacket then evolves in time, and if carefully chosen may develop favourable Franck-Condon overlap with lowlying vibrational levels of the electronic ground state.

Vala et al. 132 have simulated the use of chirped picosecond laser pulses to form $\mathrm{Cs}_{2}$ molecules in the doubleminimum $0_{g}^{-}$state, and Luc-Koenig et al. [133, 134] have investigated optimization of the pulse characteristics. Koch et al. [135] have simulated two-photon photoassociation using the scheme shown in Fig. 11 and optimized the parameters of the dump pulse to maximize the formation of molecules in deeply bound vibrational states. Salzmann et al. 136 have carried out initial experimental work in which evolutionary strategies are used to optimize pulse parameters to maximise formation of ultracold ${ }^{85} \mathrm{Rb}_{2}$, while Brown et al. [137] have found that chirped femtosecond pulses produce fewer ultracold ${ }^{85} \mathrm{Rb}_{2}$ and ${ }^{87} \mathrm{Rb}_{2}$ molecules than comparable unchirped pulses.

\section{Molecules in low vibrational states}

Both photoassociation and magnetic resonance tuning produce molecules in very high vibrational states. However, molecules in excited vibrational states can always undergo inelastic collisions that lead to trap loss. There is thus great interest in finding ways either to drive the formation of molecules in the vibrational ground state, $v=0$, or to transfer molecules initially formed in highlying states to $v=0$.

Direct photoassociation to form low-lying vibrational states is not usually feasible for homonuclear molecules, because the low-energy scattering wavefunction for a pair of atoms has very little amplitude at short range. There is therefore very little Franck-Condon overlap with the wavefunctions for low-lying vibrational states, which are entirely at short range. Various schemes have been proposed to overcome this 19, 138, 139], but for homonuclear molecules the combination of parity restrictions and Franck-Condon factors present formidable obstacles. For

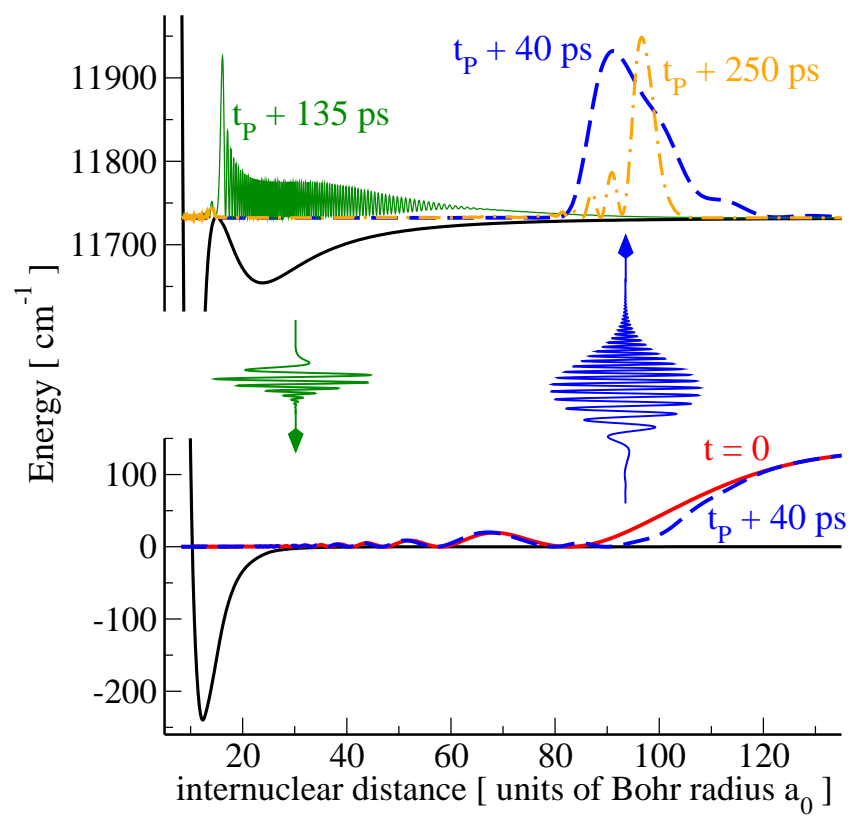

FIG. 11: Formation of $\mathrm{Cs}_{2}$ using chirped pump and dump pulses, showing the evolution of the wavepacket on the upper electronic state $\left(0_{g}^{-}\right)$. Reprinted with permission from Koch et al. [135]. Copyright 2006 by the American Physical Society.

example, Jaksch et al. 140] proposed a 6-photon scheme to form ${ }^{87} \mathrm{Rb}_{2}$ in its ground vibronic state. For heteronuclear species, on the other hand, the parity restrictions are lifted and the Franck-Condon factors are more favourable 141], so that stimulated Raman photoassociation to form $v=0$ molecules may be feasible [19, 139].

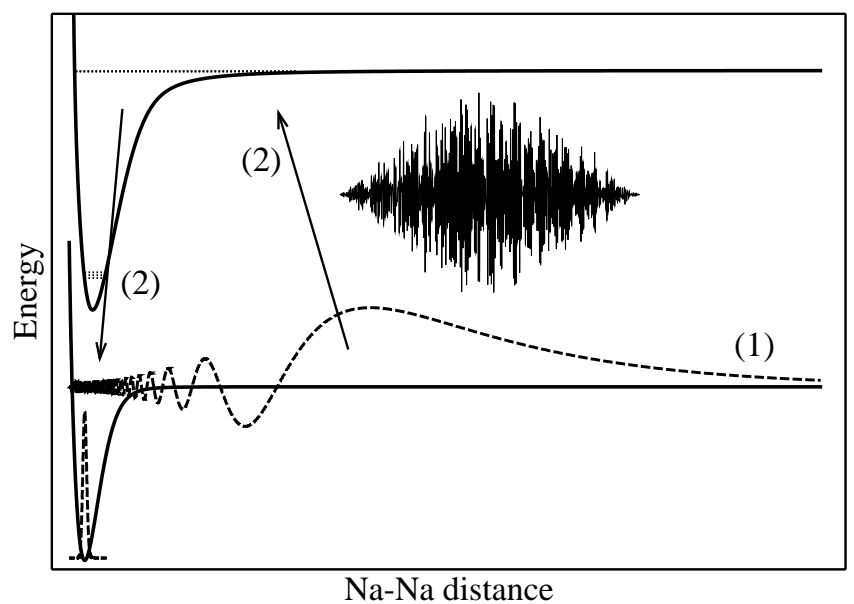

FIG. 12: The use of tailored laser pulses to stabilise longrange $\mathrm{Na}_{2}$ molecules by transferring them to the ground vibronic state. Reprinted with permission from Koch et al. 142]. Copyright 2004 by the American Physical Society.

An alternative approach that may be advantageous is to form long-range molecules first, by either Feshbach 
resonance tuning or photoassociation, and then transfer the molecular population to a short-range state. Koch et al. 142 have used optimal control theory to design tailored laser pulses that would achieve this for $\mathrm{Na}_{2}$ as shown in Fig. 12] while Stwalley [143] has suggested that for heteronuclear alkali metal dimers it could be efficiently achieved by stimulated Raman adiabatic passage via mixed levels of the $\mathrm{b}^{3} \Pi$ and $\mathrm{A}^{1} \Sigma^{+}$states.

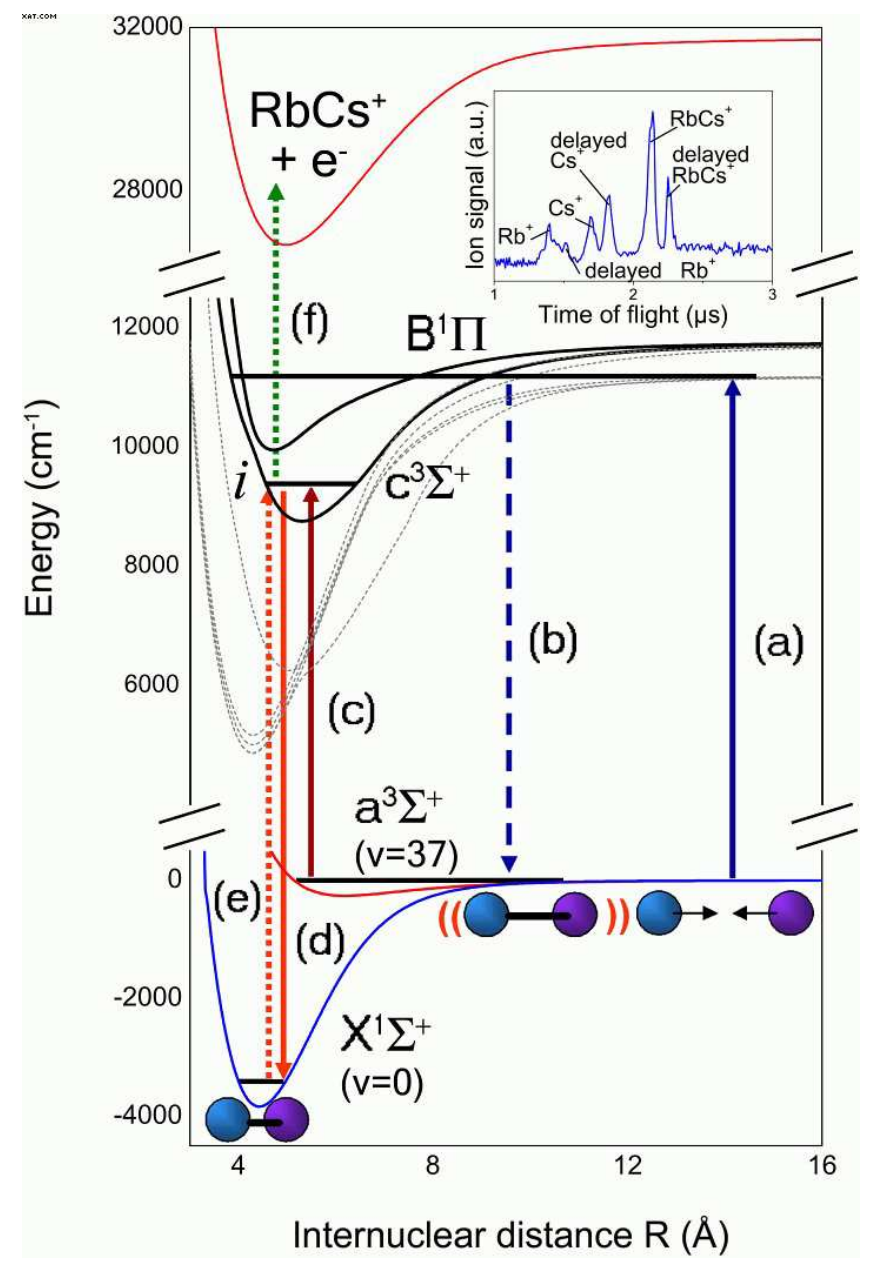

FIG. 13: The scheme used to produce and detect RbCs molecules in their ground vibronic state. Reprinted with permission from Sage et al. [144]. Copyright 2005 by the American Physical Society.

In the culmination of a series of papers 145, 146, 147], Sage et al. [144] have succeeded in creating ultracold RbCs molecules $(T \approx 100 \mu \mathrm{K})$ in their vibronic ground state using the 2-step (4-photon) process shown in Fig. 13 first, molecules are produced in the weakly bound $v=37$ level of the $\mathrm{a}^{3} \Sigma^{+}$state by 1 -photon photoassociation (a) followed by spontaneous emission (b), and then they are transferred to the $v=0$ or 1 level of the $\mathrm{X}^{1} \Sigma^{+}$state by an incoherent 2-photon pump/dump process (stimulated emission pumping, SEP, (c) and (d) in Fig. 13) via a mixed level of the $c$ and $B$ excited states.
The SEP process has an efficiency of only $6 \%$, but this could in principle be dramatically improved by using STIRAP instead.

Other heteronuclear molecules such as KRb 148, 149, 150] and NaCs 151] have also been produced in the electronic ground state 148, 149, 151] and state-selectively detected [150], but not yet transferred to low-lying vibrational states. LiCs and $\mathrm{NaCs}$ have been formed in the lowest vibrational level of the lowest triplet state on the surface of helium droplets [152], but the temperature is that of the droplet $(0.38 \mathrm{~K})$ and cannot easily be lowered further.

\section{MOLECULES IN OPTICAL LATTICES}

An optical lattice is formed by standing waves between two or more laser beams. Since atoms and molecules are polarisable, they experience a periodic potential with minima at the points where the electric fields due to the laser are greatest. The separation between successive minima is half the laser wavelength, and the heights of the barriers between minima can be adjusted by varying the laser intensity. Confinement of atoms in optical lattice cells was first observed by Westbrook et al. 153]. One particularly interesting state that can be created is a Mott insulator phase [154, 155], in which the lattice sites are occupied in a regular pattern and which can be "melted" to form a superfluid by lowering the barriers. The dynamics of Bose-Einstein condensates in optical lattices have been recently reviewed by Morsch and Oberthaler [156].

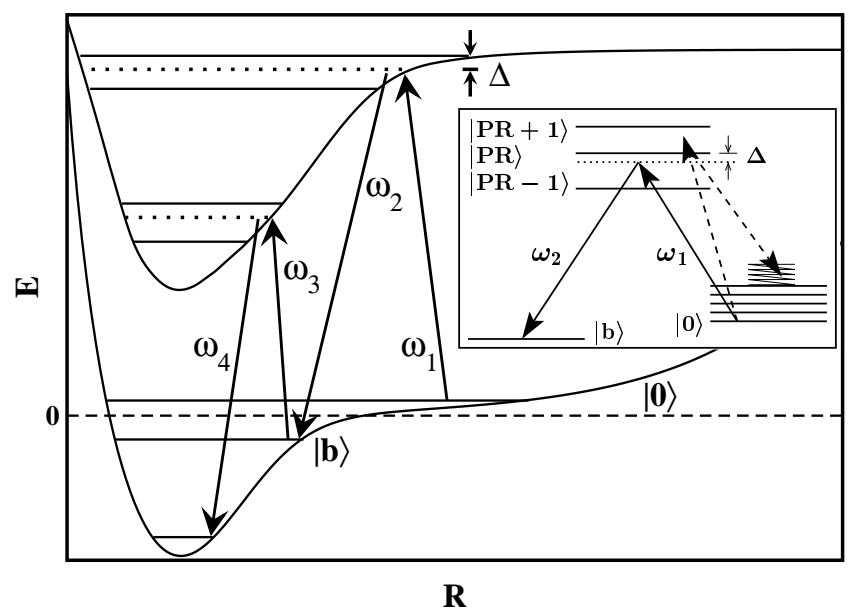

FIG. 14: Scheme for creating molecules at doubly occupied lattice sites in a Mott insulator. Note the quadratic trapping potential at long range that serves to confine the initial state. Reprinted with permission from Jaksch et al. [140]. Copyright 2002 by the American Physical Society.

The production of molecules in optical lattices offers intriguing possibilities. The barriers between lat- 
tice sites enhance stability by preventing collisions between molecules at different sites. Jaksch et al. 140 proposed creating a molecular Bose-Einstein condensate by Raman photoassociation in a Mott insulator with 2 atoms in each lattice site as shown in Fig. 14. The trapping potential discretizes the molecular continuum and converts the free-bound photoassociation process into a bound-bound transition. Damski et al. [157] extended this idea to the creation of a dipolar superfluid by photoassociation in a lattice containing one atom of each of two different species in each site. Rom et al. 158] have created ${ }^{87} \mathrm{Rb}_{2}$ molecules in an optical lattice by essentially the technique of ref. 140. Ryu et al. [159] have carried out similar experiments and observed coherent oscillations (Rabi cycling) between atomic and molecular gases. They also observed distinct Raman spectra for photoassociation occurring in doubly and triply occupied sites. Thalhammer et al. 160 have also created ${ }^{87} \mathrm{Rb}_{2}$ on an optical lattice, but by magnetic tuning through a Feshbach resonance. They were able to purify the resulting molecular lattice by driving out the remaining atoms with a laser, and showed that the molecules remained trapped much longer $(700 \mathrm{~ms})$ after the atoms had been removed than while they were still present. They attribute the loss rate to inelastic atom-molecule collisions that occur when atoms tunnel through lattice barriers into sites occupied by molecules; once the atoms are removed, the corresponding loss due to molecule-molecule collisions is much slower simply because of the larger mass and reduced tunnelling rate of the molecules. Very recently, Winkler et al. 161 have created long-lived bound atom pairs with a repulsive atom-atom interaction. The pairs cannot decay into separated atoms because the energies of the atoms that would be produced are not allowed by the lattice band structure. Remarkably, the pairs do decay if the repulsive interaction between the atoms is switched off by tuning the scattering length to zero. Volz et al. [162] have created a Mott state of ${ }^{87} \mathrm{Rb}_{2}$ molecules and demonstrated that phase coherence is restored when the lattice depth is reduced.

Initial experiments have also been carried out on fermion dimers in optical lattices. Moritz et al. 163] reported the creation of a 1-dimensional gas of ${ }^{40} K_{2}$ molecules in a 2-d optical lattice by Feshbach resonance tuning, and Stöferle et al. [164] have carried out similar experiments in a 3-d lattice. However, the tunnelling rates for the lattices used were too fast for lifetimes to be increased beyond those normal for fermion dimers.

Optical lattices provide a very promising environment for bringing together more than two atoms at a time and studying many-body processes under controlled conditions. Stoll and Köhler 165] have suggested a scheme that could be used to produce Efimov states of alkali metal trimers directly from 3 atoms by magnetic Feshbach resonance tuning in an optical lattice.

\section{COLLISIONS OF ULTRACOLD MOLECULES}

Collision processes involving ultracold molecules are of prime importance to trapping and controlling them. As described above, inelastic collisions usually release enough kinetic energy that both collision partners are lost from the trap. The initial experiments on boson dimers formed by Feshbach resonance tuning 67, 68, 69, 70] gave lifetimes that suggested vibrational relaxation rates for atom-molecule collisions around $10^{-10} \mathrm{~cm}^{3} \mathrm{~s}^{-1}$. Quantitative estimations were not usually attempted, but this was consistent with the rate of $1.6 \times 10^{-10} \mathrm{~cm}^{3} \mathrm{~s}^{-1}$, estimated by Yurovsky et al. [166] on the basis of early Feshbach resonance experiments [59,60]. It also agreed with quantum dynamics calculations by Soldan et al. 167] on vibrational relaxation in $\mathrm{Na}+\mathrm{Na}_{2}$ collisions. More recently, Mukaiyama et al. [168] have measured the trap loss rate for ${ }^{23} \mathrm{Na}_{2}$ molecules formed by Feshbach resonance tuning and obtained an atom-molecule rate coefficient $k_{\text {loss }}=5.1 \times 10^{-11} \mathrm{~cm}^{3} \mathrm{~s}^{-1}$ for molecules in the highest vibrational state.

Relaxation processes involving molecules formed by photoassociation have also been studied. Wynar et al. 119] obtained an upper bound of $k_{\text {loss }}=8 \times 10^{-11}$ $\mathrm{cm}^{3} \mathrm{~s}^{-1}$ for ${ }^{87} \mathrm{Rb}_{2}$ molecules in the second-to-last vibrationally excited state. Staanum et al. 169] have investigated inelastic collisions of rovibrationally excited $\mathrm{Cs}_{2}$ $\left({ }^{3} \Sigma_{u}^{+}\right)$in collisions with Cs atoms in two different ranges of the vibrational quantum number $v$ by monitoring trap loss of $\mathrm{Cs}_{2}$. They obtained atom-molecule rate coefficients close to $1.0 \times 10^{-10} \mathrm{~cm}^{3} \mathrm{~s}^{-1}$ for both $v=4$ to 6 and $v=32$ to 47 . Zahzam et al. 170 have carried out similar work for different rovibrational states of ${ }^{3} \Sigma_{u}^{+}$, but also considered molecules in the ${ }^{1} \Sigma_{g}^{+}$state and moleculemolecule collisions. They obtained rate coefficients of $2.6 \times 10^{-11} \mathrm{~cm}^{3} \mathrm{~s}^{-1}$ and $1.0 \times 10^{-11} \mathrm{~cm}^{3} \mathrm{~s}^{-1}$ in the atom-atom and atom-molecule cases respectively, both with quite large error bounds.

Soldán et al. 167], Quéméner et al. 171, 172], and Cvitaš et al. [84, 173, 174] have carried out quantum dynamics calculations on atom-molecule collisions between alkali-metal atoms and dimers. This work will be described in greater detail in a forthcoming review [175]. The calculations used a reactive scattering approach developed by Launay and LeDourneuf [176], which has been applied extensively to chemical reactions such as $\mathrm{N}\left({ }^{2} \mathrm{D}\right)+\mathrm{H}_{2}$ [177] and $\mathrm{O}\left({ }^{1} \mathrm{D}\right)+\mathrm{H}_{2}$ [178] at higher energies. Soldán et al. 167] showed that barrierless atom exchange reactions can occur in $\mathrm{Na}+\mathrm{Na}_{2}$, and that even at very low energy such collisions cause very fast vibrational relaxation ( $k_{\text {inel }}$ on the order of $10^{-10} \mathrm{~cm}^{3} \mathrm{~s}^{-1}$ ) for collisions of molecules in low vibrational states. The cross sections were shown to depend strongly on the details of the potential energy surfaces, and to change by a factor of 10 when non-additive forces were included, 

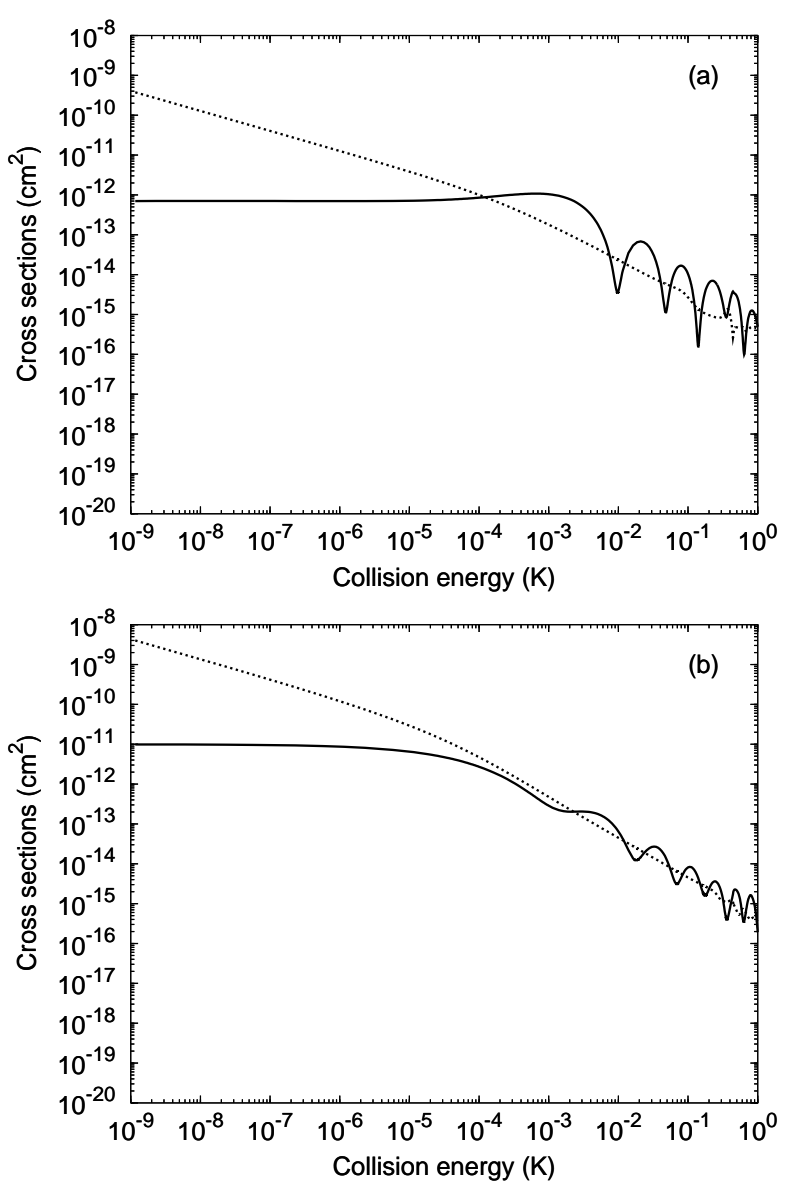

FIG. 15: Cross sections from s-wave quantum reactive scattering calculations for $\mathrm{Na}+\mathrm{Na}_{2}(v=1, j=0)$ Elastic and quenching results are shown as solid and dotted lines. The cross sections on the additive potential (a) are a factor of 10 smaller than those on the non-additive potential (b). Reproduced from Soldán et al. [167]

as shown in Fig. [15] In subsequent work, Soldán et al. [179] showed that non-additive forces are important for all the alkali metal trimer systems, and affect the well depth for spin-polarized $\mathrm{Li}+\mathrm{Li}_{2}$ collisions by a factor of 4 . The quantum dynamics calculations were subsequently extended to $\mathrm{Li}+\mathrm{Li}_{2}$ collisions, both isotopically homonuclear [84, 173] and heteronuclear 174], and to K $+\mathrm{K}_{2}$ [172]. For the homonuclear Li systems, Cvitaš et al. 84] demonstrated that there is no systematic suppression of the inelastic collision rates for fermion dimers in low vibrational states, in contrast to the situation for molecules in long-range states [75, 76, 77, 78, 82, 83]. This is very important for attempts to transfer molecules formed by Feshbach resonance tuning to the vibrational ground state, because it means that the transfer must be accomplished without spending significant time in intermediate vibrational states.

\section{CONCLUSIONS}

There have been enormous advances in the production and manipulation of molecules in laser-cooled atomic gases. Molecules have been produced both by photoassociation and by magnetic tuning through Feshbach resonances. Molecular Bose-Einstein condensates have been produced for molecules in long-range states, and the first signatures of triatomic and tetraatomic molecules have been seen. Most of the experimental advances were guided by theoretical predictions, and the experiments have in turn stimulated a large number of theoretical studies.

Prospects for the future include the use of cold molecules for high-precision measurement and the production of quantum-degenerate gases of ground-state molecules, which will be stable to collisions and offer a wealth of new possibilities for quantum control. Heteronuclear molecules are particularly interesting, because they can have substantial dipole moments in long-range states. Dipolar quantum gases offer a new range of novel properties, and ultracold polar molecules also have potential applications in quantum computing and in studying fundamental physical properties such as parity violation and the electron dipole moment.

\section{ACKNOWLEDGMENTS}

The authors are grateful to Simon Cornish and Charles Adams for valuable discussions and to Eite Tiesinga and Cheng Chin for providing Figures based on unpublished work. PS acknowledges support from the Ministry of Education of the Czech Republic (grant no. LC06002).

[1] M. H. Anderson, J. R. Ensher, M. R. Matthews, C. E. Wieman, and E. A. Cornell, Science 269, 198 (1995).

[2] C. C. Bradley, C. A. Sackett, J. J. Tollett, and R. G. Hulet, Phys. Rev. Lett. 75, 1687 (1995).

[3] K. B. Davis, M. O. Mewes, M. R. Andrews, N. J. VanDruten, D. S. Durfee, D. M. Kurn, and W. Ketterle, Phys. Rev. Lett. 75, 3969 (1995).

[4] S. L. Cornish, N. R. Claussen, J. L. Roberts, E. A. Cornell, and C. E. Wieman, Phys. Rev. Lett. 85, 1795 (2000).

[5] G. Modugno, G. Ferrari, G. Roati, R. J. Brecha, A. Simoni, and M. Inguscio, Science 294, 1320 (2001).

[6] T. Weber, J. Herbig, M. Mark, H. C. Nägerl, and R. Grimm, Science 299, 232 (2003).

[7] D. G. Fried, T. C. Killian, L. Willmann, D. Landhuis, S. C. Moss, D. Kleppner, and T. J. Greytak, Phys. Rev. Lett. 81, 3811 (1998).

[8] A. Robert, O. Sirjean, A. Browaeys, J. Poupard, S. Nowak, D. Boiron, C. I. Westbrook, and A. Aspect, Science 292, 461 (2001). 
[9] F. P. Dos Santos, J. Leonard, J. M. Wang, C. J. Barrelet, F. Perales, E. Rasel, C. S. Unnikrishnan, M. Leduc, and C. Cohen-Tannoudji, Phys. Rev. Lett. 86, 3459 (2001).

[10] Y. Takasu, K. Maki, K. Komori, T. Takano, K. Honda, M. Kumakura, T. Yabuzaki, and Y. Takahashi, Phys. Rev. Lett. 91, 040404 (2003).

[11] A. Griesmaier, J. Werner, S. Hensler, J. Stuhler, and T. Pfau, Phys. Rev. Lett. 94, 160401 (2005).

[12] E. A. Cornell and C. E. Wieman, Rev. Mod. Phys. 74, 875 (2002).

[13] W. Ketterle, Rev. Mod. Phys. 74, 1131 (2002).

[14] C. J. Pethick and H. Smith, Bose-Einstein Condensation in Dilute Gases (Cambridge University Press, 2002).

[15] S. L. Cornish and D. Cassettari, Philos. Trans. R. Soc. Lond. Ser. A-Math. Phys. Eng. Sci. 361, 2699 (2003).

[16] B. DeMarco and D. S. Jin, Science 285, 1703 (1999).

[17] A. G. Truscott, K. E. Strecker, W. I. McAlexander, G. B. Partridge, and R. G. Hulet, Science 291, 2570 (2001).

[18] M. Baranov, Ł. Dobrek, K. Góral, L. Santos, and M. Lewenstein, Phys. Scr. T102, 74 (2002).

[19] D. DeMille, Phys. Rev. Lett. 88, 067901 (2002).

[20] J. J. Hudson, B. E. Sauer, M. R. Tarbutt, and E. A. Hinds, Phys. Rev. Lett. 89, 023003 (2002).

[21] M. Quack and J. Stohner, Chimia 59, 530 (2005).

[22] J. Crassous, C. Chardonnet, T. Saue, and P. Schwerdtfeger, Org. Biomol. Chem. 3, 2218 (2005).

[23] E. R. Hudson, H. J. Lewandowski, B. C. Sawyer, and J. Ye, Phys. Rev. Lett. 96, 143004 (2006).

[24] H. L. Bethlem and G. Meijer, Int. Rev. Phys. Chem. 22, 73 (2003).

[25] R. V. Krems, Int. Rev. Phys. Chem. 24, 99 (2005).

[26] E. Bodo and F. A. Gianturco, Int. Rev. Phys. Chem. 25, 313 (2006).

[27] P. F. Weck and N. Balakrishnan, Int. Rev. Phys. Chem. 25, 283 (2006).

[28] C. S. Adams and E. Riis, Prog. Quantum Electron. 21, 1 (1997).

[29] S. Chu, Rev. Mod. Phys. 70, 685 (1998).

[30] W. D. Phillips, Rev. Mod. Phys. 70, 721 (1998).

[31] C. N. Cohen-Tannoudji, Rev. Mod. Phys. 70, 707 (1998).

[32] E. Tiesinga (2006), private communication.

[33] W. D. Phillips and H. Metcalf, Phys. Rev. Lett. 48, 596 (1982).

[34] A. L. Migdall, J. V. Prodan, W. D. Phillips, T. H. Bergeman, and H. J. Metcalf, Phys. Rev. Lett. 54, 2596 (1985).

[35] E. L. Raab, M. Prentiss, A. Cable, S. Chu, and D. E. Pritchard, Phys. Rev. Lett. 59, 2631 (1987).

[36] S. Chu, L. Hollberg, J. E. Bjorkholm, A. Cable, and A. Ashkin, Phys. Rev. Lett. 55, 48 (1985).

[37] J. Dalibard and C. Cohen-Tannoudji, J. Opt. Soc. Am. B-Opt. Phys. 6, 2023 (1989).

[38] P. D. Lett, R. N. Watts, C. I. Westbrook, W. D. Phillips, P. L. Gould, and H. J. Metcalf, Phys. Rev. Lett. 61, 169 (1988).

[39] N. Masuhara, J. M. Doyle, J. C. Sandberg, D. Kleppner, T. J. Greytak, H. F. Hess, and G. P. Kochanski, Phys. Rev. Lett. 61, 935 (1988).

[40] S. Chu, J. E. Bjorkholm, A. Ashkin, and A. Cable, Phys. Rev. Lett. 57, 314 (1986).
[41] J. D. Miller, R. A. Cline, and D. J. Heinzen, Phys. Rev. A 47, R4567 (1993).

[42] T. Takekoshi and R. J. Knize, Opt. Lett. 21, 77 (1996).

[43] T. Takekoshi, B. M. Patterson, and R. J. Knize, Phys. Rev. Lett. 81, 5105 (1998).

[44] J. D. Weinstein, R. deCarvalho, T. Guillet, B. Friedrich, and J. M. Doyle, Nature 395, 148 (1998).

[45] N. Vanhaecke, W. D. Melo, B. L. Tolra, D. Comparat, and P. Pillet, Phys. Rev. Lett. 89, 063001 (2002).

[46] H. L. Bethlem, G. Berden, F. M. H. Crompvoets, R. T. Jongma, A. J. A. van Roij, and G. Meijer, Nature 406, 491 (2000).

[47] Y. Kagan, G. V. Shlyapnikov, and J. T. M. Walraven, Phys. Rev. Lett. 76, 2670 (1996).

[48] C. C. Bradley, C. A. Sackett, and R. G. Hulet, Phys. Rev. Lett. 78, 985 (1997).

[49] E. Tiesinga, B. J. Verhaar, and H. T. C. Stoof, Phys. Rev. A 47, 4114 (1993).

[50] H. Feshbach, Ann. Phys. 5, 357 (1958).

[51] P. Courteille, R. S. Freeland, D. J. Heinzen, F. A. van Abeelen, and B. J. Verhaar, Phys. Rev. Lett. 81, 69 (1998).

[52] A. Marte, T. Volz, J. Schuster, S. Durr, G. Rempe, E. G. M. van Kempen, and B. J. Verhaar, Phys. Rev. Lett. 89, 283202 (2002).

[53] A. J. Moerdijk, B. J. Verhaar, and A. Axelsson, Phys. Rev. A 51, 4852 (1995).

[54] T. Weber, J. Herbig, M. Mark, H. C. Nägerl, and R. Grimm, Science 299, 232 (2003).

[55] C. Chin, V. Vuletić, A. J. Kerman, S. Chu, E. Tiesinga, P. J. Leo, and C. J. Williams, Phys. Rev. A 70, 032701 (2004)

[56] C. Chin, A. J. Kerman, V. Vuletić, and S. Chu, Phys. Rev. Lett. 90, 033201 (2003).

[57] C. Chin (2006), private communication.

[58] C. J. Joachain, Quantum Collision Theory (NorthHolland, Amsterdam, 1975).

[59] S. Inouye, M. R. Andrews, J. Stenger, H. J. Miesner, D. M. Stamper-Kurn, and W. Ketterle, Nature 392, 151 (1998).

[60] J. Stenger, S. Inouye, M. R. Andrews, H. J. Miesner, D. M. Stamper-Kurn, and W. Ketterle, Phys. Rev. Lett. 82, 2422 (1999).

[61] F. A. van Abeelen and B. J. Verhaar, Phys. Rev. Lett. 83, 1550 (1999).

[62] F. H. Mies, E. Tiesinga, and P. S. Julienne, Phys. Rev. A 61, 022721 (2000).

[63] V. A. Yurovsky, A. Ben-Reuven, P. S. Julienne, and C. J. Williams, Phys. Rev. A 60, R765 (1999).

[64] J. L. Roberts, N. R. Claussen, S. L. Cornish, E. A. Donley, E. A. Cornell, and C. E. Wieman, Phys. Rev. Lett. 86, 4211 (2001).

[65] E. A. Donley, N. R. Claussen, S. L. Cornish, J. L. Roberts, E. A. Cornell, and C. E. Wieman, Nature 412, 295 (2001).

[66] E. Timmermans, P. Tommasini, M. Hussein, and A. Kerman, Phys. Rep.-Rev. Sec. Phys. Lett. 315, 199 (1999).

[67] J. Herbig, T. Kraemer, M. Mark, T. Weber, C. Chin, H. C. Nägerl, and R. Grimm, Science 301, 1510 (2003).

[68] E. A. Donley, N. R. Claussen, S. T. Thompson, and C. E. Wieman, Nature 417, 529 (2002).

[69] S. Dürr, T. Volz, A. Marte, and G. Rempe, Phys. Rev. Lett. 92, 020406 (2004). 
[70] K. Xu, T. Mukaiyama, J. R. Abo-Shaeer, J. K. Chin, D. E. Miller, and W. Ketterle, Phys. Rev. Lett. 91, 210402 (2003).

[71] S. T. Thompson, E. Hodby, and C. E. Wieman, Phys. Rev. Lett. 94, 020401 (2005).

[72] T. Köhler, E. Tiesinga, and P. S. Julienne, Phys. Rev. Lett. 94, 020402 (2005).

[73] T. Köhler, T. Gasenzer, P. S. Julienne, and K. Burnett, Phys. Rev. Lett. 91, 230401 (2003).

[74] C. A. Regal, C. Ticknor, J. L. Bohn, and D. S. Jin, Nature 424, 47 (2003).

[75] K. E. Strecker, G. B. Partridge, and R. G. Hulet, Phys. Rev. Lett. 91, 080406 (2003).

[76] J. Cubizolles, T. Bourdel, S. J. J. M. F. Kokkelmans, G. V. Shlyapnikov, and C. Salomon, Phys. Rev. Lett. 91, 240401 (2003).

[77] S. Jochim, M. Bartenstein, A. Altmeyer, G. Hendl, C. Chin, J. H. Denschlag, and R. Grimm, Phys. Rev. Lett. 91, 240402 (2003).

[78] C. A. Regal, M. Greiner, and D. S. Jin, Phys. Rev. Lett. 92, 083201 (2004).

[79] S. Jochim, M. Bartenstein, A. Altmeyer, G. Hendl, S. Riedl, C. Chin, J. H. Denschlag, and R. Grimm, Science 302, 2101 (2003).

[80] M. W. Zwierlein, C. A. Stan, C. H. Schunck, S. M. F. Raupach, S. Gupta, Z. Hadzibabic, and W. Ketterle, Phys. Rev. Lett. 91, 250401 (2003).

[81] M. Greiner, C. A. Regal, and D. S. Jin, Nature 426, 537 (2003).

[82] D. S. Petrov, C. Salomon, and G. V. Shlyapnikov, Phys. Rev. Lett. 93, 090404 (2004).

[83] D. S. Petrov, C. Salomon, and G. V. Shlyapnikov, Phys. Rev. A 71, 012708 (2005).

[84] M. T. Cvitaš, P. Soldán, J. M. Hutson, P. Honvault, and J. M. Launay, Phys. Rev. Lett. 94, 033201 (2005).

[85] J. Bardeen, L. N. Cooper, and J. R. Schrieffer, 108, 1175 (1957).

[86] M. Bartenstein, A. Altmeyer, S. Riedl, S. Jochim, C. Chin, J. H. Denschlag, and R. Grimm, Phys. Rev. Lett. 92, 120401 (2004).

[87] C. A. Regal, M. Greiner, and D. S. Jin, Phys. Rev. Lett. 92, 040403 (2004).

[88] C. A. Regal, M. Greiner, S. Giorgini, M. Holland, and D. S. Jin, Phys. Rev. Lett. 95, 250404 (2005).

[89] M. W. Zwierlein, C. A. Stan, C. H. Schunck, S. M. F. Raupach, A. J. Kerman, and W. Ketterle, Phys. Rev. Lett. 92, 120403 (2004).

[90] C. Chin, M. Bartenstein, A. Altmeyer, S. Riedl, S. Jochim, J. H. Denschlag, and R. Grimm, Science 305, 1128 (2004).

[91] M. Greiner, C. A. Regal, and D. S. Jin, Phys. Rev. Lett. 94, 070403 (2005).

[92] G. B. Partridge, K. E. Strecker, R. I. Kamar, M. W. Jack, and R. G. Hulet, Phys. Rev. Lett. 95, 020404 (2005).

[93] J. Kinast, S. L. Hemmer, M. E. Gehm, A. Turlapov, and J. E. Thomas, Phys. Rev. Lett. 92, 150402 (2004).

[94] M. Bartenstein, A. Altmeyer, S. Riedl, S. Jochim, C. Chin, J. H. Denschlag, and R. Grimm, Phys. Rev. Lett. 92, 203201 (2004).

[95] J. Kinast, A. Turlapov, J. E. Thomas, Q. J. Chen, J. Stajic, and K. Levin, Science 307, 1296 (2005).

[96] M. W. Zwierlein, J. R. Abo-Shaeer, A. Schirotzek, C. H. Schunck, and W. Ketterle, Nature 435, 1047 (2005).
[97] S. Inouye, J. Goldwin, M. L. Olsen, C. Ticknor, J. L. Bohn, and D. S. Jin, Phys. Rev. Lett. 93, 183201 (2004).

[98] F. Ferlaino, C. D'Errico, G. Roati, M. Zaccanti, M. Inguscio, G. Modugno, and A. Simoni, Phys. Rev. A 73, 040702 (2006).

[99] C. A. Stan, M. W. Zwierlein, C. H. Schunck, S. M. F. Raupach, and W. Ketterle, Phys. Rev. Lett. 93, 143001 (2004).

[100] M. Aymar and O. Dulieu, J. Chem. Phys. 122, 204302 (2005).

[101] W. B. Brown and D. M. Whisnant, Mol. Phys. 25, 1385 (1973).

[102] C. Chin, T. Kraemer, M. Mark, J. Herbig, P. Waldburger, H. C. Nägerl, and R. Grimm, Phys. Rev. Lett. 94, 123201 (2005).

[103] T. Kraemer, M. Mark, P. Waldburger, J. G. Danzl, C. Chin, B. Engeser, A. D. Lange, K. Pilch, A. Jaakkola, H. C. Nägerl, et al., Nature 440, 315 (2006).

[104] V. Efimov, Phys. Lett. B 33, 563 (1970).

[105] T. K. Lim, K. Duffy, and W. C. Damert, Phys. Rev. Lett. 38, 341 (1977).

[106] B. D. Esry, C. D. Lin, and C. H. Greene, Phys. Rev. A 54, 394 (1996).

[107] E. Braaten and H. W. Hammer, Phys. Rev. Lett. 8716, 160407 (2001).

[108] E. Nielsen, H. Suno, and B. D. Esry, Phys. Rev. A 66, 012705 (2002).

[109] H. R. Thorsheim, J. Weiner, and P. S. Julienne, Phys. Rev. Lett. 58, 2420 (1987).

[110] W. C. Stwalley and H. Wang, J. Mol. Spectrosc. 195, 194 (1999).

[111] K. M. Jones, E. Tiesinga, P. D. Lett, and P. S. Julienne, Rev. Mod. Phys. 78, 483 (2006).

[112] A. Fioretti, D. Comparat, A. Crubellier, O. Dulieu, F. Masnou-Seeuws, and P. Pillet, Phys. Rev. Lett. 80, 4402 (1998).

[113] A. Fioretti, D. Comparat, C. Drag, C. Amiot, O. Dulieu, F. Masnou-Seeuws, and P. Pillet, Eur. Phys. J. D 5, 389 (1999).

[114] A. N. Nikolov, E. E. Eyler, X. T. Wang, J. Li, H. Wang, W. C. Stwalley, and P. L. Gould, Phys. Rev. Lett. 82, 703 (1999).

[115] A. N. Nikolov, J. R. Ensher, E. E. Eyler, H. Wang, W. C. Stwalley, and P. L. Gould, Phys. Rev. Lett. 84, 246 (2000).

[116] H. M. J. M. Boesten, C. C. Tsai, D. J. Heinzen, A. J. Moonen, and B. J. Verhaar, J. Phys. B-At. Mol. Opt. Phys. 32, 287 (1999).

[117] U. Gaubatz, P. Rudecki, S. Schiemann, and K. Bergmann, J. Chem. Phys. 92, 5363 (1990).

[118] P. S. Julienne, K. Burnett, Y. B. Band, and W. C. Stwalley, Phys. Rev. A 58, R797 (1998).

[119] R. Wynar, R. S. Freeland, D. J. Han, C. Ryu, and D. J. Heinzen, Science 287, 1016 (2000).

[120] D. J. Heinzen, R. Wynar, P. D. Drummond, and K. V. Kheruntsyan, Phys. Rev. Lett. 84, 5029 (2000).

[121] P. D. Drummond, K. V. Kheruntsyan, D. J. Heinzen, and R. H. Wynar, Phys. Rev. A 65, 063619 (2002).

[122] K. Winkler, G. Thalhammer, M. Theis, H. Ritsch, R. Grimm, and J. H. Denschlag, Phys. Rev. Lett. 95, 063202 (2005).

[123] S. T. Thompson, E. Hodby, and C. E. Wieman, Phys. Rev. Lett. 95, 190404 (2005).

[124] P. O. Fedichev, Y. Kagan, G. V. Shlyapnikov, and 
J. T. M. Walraven, Phys. Rev. Lett. 77, 2913 (1996).

[125] J. L. Bohn and P. S. Julienne, Phys. Rev. A 56, 1486 (1997).

[126] F. K. Fatemi, K. M. Jones, and P. D. Lett, Phys. Rev. Lett. 85, 4462 (2000).

[127] M. Theis, G. Thalhammer, K. Winkler, M. Hellwig, G. Ruff, R. Grimm, and J. H. Denschlag, Phys. Rev. Lett. 93, 123001 (2004).

[128] G. Thalhammer, M. Theis, K. Winkler, R. Grimm, and J. H. Denschlag, Phys. Rev. A 71, 033403 (2005).

[129] J. Javanainen and M. Mackie, Phys. Rev. A 59, R3186 (1999).

[130] C. P. Koch, F. Masnou-Seeuws, and R. Kosloff, Phys. Rev. Lett. 94, 193001 (2005).

[131] M. Dantus and V. V. Lozovoy, Chem. Rev. 104, 1813 (2004).

[132] J. Vala, O. Dulieu, F. Masnou-Seeuws, P. Pillet, and R. Kosloff, Phys. Rev. A 63, 013412 (2001).

[133] E. Luc-Koenig, R. Kosloff, F. Masnou-Seeuws, and M. Vatasescu, Phys. Rev. A 70, 033414 (2004).

[134] E. Luc-Koenig, M. Vatasescu, and F. Masnou-Seeuws, Eur. Phys. J. D 31, 239 (2004).

[135] C. P. Koch, E. Luc-Koenig, and F. Masnou-Seeuws, Phys. Rev. A 73, 033408 (2006).

[136] W. Salzmann, U. Poschinger, R. Wester, M. Weidemüller, A. Merli, S. M. Weber, F. Sauer, M. Plewicki, F. Weise, A. M. Esparza, et al., Phys. Rev. A 73, 023414 (2006).

[137] B. L. Brown, A. J. Dicks, and I. A. Walmsley, Phys. Rev. Lett. 96, 173002 (2006).

[138] Y. B. Band and P. S. Julienne, Phys. Rev. A 51, R4317 (1995).

[139] S. Kotochigova, E. Tiesinga, and P. S. Julienne, Eur. Phys. J. D 31, 189 (2004).

[140] D. Jaksch, V. Venturi, J. I. Cirac, C. J. Williams, and P. Zoller, Phys. Rev. Lett. 89, 040402 (2002).

[141] H. Wang and W. C. Stwalley, J. Chem. Phys. 108, 5767 (1998).

[142] C. P. Koch, J. P. Palao, R. Kosloff, and F. MasnouSeeuws, Phys. Rev. A 70, 013402 (2004).

[143] W. C. Stwalley, Eur. Phys. J. D 31, 221 (2004).

[144] J. M. Sage, S. Sainis, T. Bergeman, and D. DeMille, Phys. Rev. Lett. 94, 203001 (2005).

[145] A. J. Kerman, J. M. Sage, S. Sainis, T. Bergeman, and D. DeMille, Phys. Rev. Lett. 92, 033004 (2004).

[146] A. J. Kerman, J. M. Sage, S. Sainis, T. Bergeman, and D. DeMille, Phys. Rev. Lett. 92, 153001 (2004).

[147] T. Bergeman, A. J. Kerman, J. Sage, S. Sainis, and D. DeMille, Eur. Phys. J. D 31, 179 (2004).

[148] M. W. Mancini, G. D. Telles, A. R. L. Caires, V. S. Bagnato, and L. G. Marcassa, Phys. Rev. Lett. 92, 133203 (2004).

[149] D. Wang, J. Qi, M. F. Stone, O. Nikolayeva, B. Hattaway, S. D. Gensemer, H. Wang, W. T. Zemke, P. L. Gould, E. E. Eyler, et al., Eur. Phys. J. D 31, 165 (2004).

[150] D. Wang, E. E. Eyler, P. L. Gould, and W. C. Stwalley, Phys. Rev. A 72, 032502 (2005).

[151] C. Haimberger, J. Kleinert, M. Bhattacharya, and N. P. Bigelow, Phys. Rev. A 70, 021402 (2004).

[152] N. Mudrich, O. Bunermann, F. Stienkemeier, O. Dulieu, and M. Weidemüller, Eur. Phys. J. D 31, 291 (2004).

[153] C. I. Westbrook, R. N. Watts, C. E. Tanner, S. L. Rolston, W. D. Phillips, P. D. Lett, and P. L. Gould, Phys. Rev. Lett. 65, 33 (1990).

[154] M. P. A. Fisher, P. B. Weichman, G. Grinstein, and D. S. Fisher, Phys. Rev. B 40, 546 (1989).

[155] M. Greiner, O. Mandel, T. Esslinger, T. W. Hänsch, and I. Bloch, Nature 415, 39 (2002).

[156] O. Morsch and M. Oberthaler, Rev. Mod. Phys. 78, 179 (2006).

[157] B. Damski, L. Santos, E. Tiemann, M. Lewenstein, S. Kotochigova, P. Julienne, and P. Zoller, Phys. Rev. Lett. 90, 110401 (2003).

[158] T. Rom, T. Best, O. Mandel, A. Widera, M. Greiner, T. W. Hänsch, and I. Bloch, Phys. Rev. Lett. 93, 073002 (2004).

[159] C. Ryu, X. Du, E. Yesilada, A. M. Dudarev, S. Wan, Q. Niu, and D. J. Heinzen, arXiv pp. cond-mat/0508201 (2006).

[160] G. Thalhammer, K. Winkler, F. Lang, S. Schmid, R. Grimm, and J. H. Denschlag, Phys. Rev. Lett. 96, 050402 (2006).

[161] K. Winkler, G. Thalhammer, F. Lang, R. Grimm, J. H. Denschlag, A. J. Daley, A. Kantian, H. P. Büchler, and P. Zoller, Nature 441, 853 (2006).

[162] T. Volz, N. Syassen, D. M. Bauer, E. Hansis, S. Dürr, and G. Rempe, arXiv pp. cond-mat/0605184 (2006).

[163] H. Moritz, T. Stöferle, K. Günter, M. Köhl, and T. Esslinger, Phys. Rev. Lett. 94, 210401 (2005).

[164] T. Stöferle, H. Moritz, K. Günter, M. Köhl, and T. Esslinger, Phys. Rev. Lett. 96, 030401 (2006).

[165] M. Stoll and T. Köhler, Phys. Rev. A 72, 022714 (2005).

[166] V. A. Yurovsky, A. Ben-Reuven, P. S. Julienne, and C. J. Williams, Phys. Rev. A 6204, 043605 (2000).

[167] P. Soldán, M. T. Cvitaš, J. M. Hutson, P. Honvault, and J. M. Launay, Phys. Rev. Lett. 89, 153201 (2002).

[168] T. Mukaiyama, J. R. Abo-Shaeer, K. Xu, J. K. Chin, and W. Ketterle, Phys. Rev. Lett. 92, 180402 (2004).

[169] P. Staanum, S. D. Kraft, J. Lange, R. Wester, and M. Weidemüller, Phys. Rev. Lett. 96, 023201 (2006).

[170] N. Zahzam, T. Vogt, M. Mudrich, D. Comparat, and P. Pillet, Phys. Rev. Lett. 96, 023202 (2006).

[171] G. Quéméner, P. Honvault, and J. M. Launay, Eur. Phys. J. D 30, 201 (2004).

[172] G. Quéméner, P. Honvault, J. M. Launay, P. Soldán, D. E. Potter, and J. M. Hutson, Phys. Rev. A 71, 032722 (2005).

[173] M. T. Cvitaš, P. Soldán, J. M. Hutson, P. Honvault, and J. M. Launay, in preparation (2006).

[174] M. T. Cvitaš, P. Soldán, J. M. Hutson, P. Honvault, and J. M. Launay, Phys. Rev. Lett. 94, 200402 (2005).

[175] P. Soldán and J. M. Hutson, in preparation (2007).

[176] J. M. Launay and M. LeDourneuf, Chem. Phys. Lett. 163, 178 (1989).

[177] P. Honvault and J. M. Launay, J. Chem. Phys. 111, 6665 (1999).

[178] P. Honvault and J. M. Launay, J. Chem. Phys. 114, 1057 (2001).

[179] P. Soldán, M. T. Cvitaš, and J. M. Hutson, Phys. Rev. A 67, 054702 (2003). 